\title{
A Post-translational Metabolic Switch Enables Complete Decoupling of Bacterial Growth from Biopolymer Production in Engineered Escherichia coli
}

Durante-Rodríguez, Gonzalo; de Lorenzo, Víctor; Nikel, Pablo I.

Published in:

A C S Synthetic Biology

Link to article, DOI:

10.1021/acssynbio.8b00345

Publication date:

2018

Document Version

Peer reviewed version

Link back to DTU Orbit

Citation (APA):

Durante-Rodríguez, G., de Lorenzo, V., \& Nikel, P. I. (2018). A Post-translational Metabolic Switch Enables Complete Decoupling of Bacterial Growth from Biopolymer Production in Engineered Escherichia coli. A C S Synthetic Biology, 7(11), 2686-2697. https://doi.org/10.1021/acssynbio.8b00345

\section{General rights}

Copyright and moral rights for the publications made accessible in the public portal are retained by the authors and/or other copyright owners and it is a condition of accessing publications that users recognise and abide by the legal requirements associated with these rights.

- Users may download and print one copy of any publication from the public portal for the purpose of private study or research.

- You may not further distribute the material or use it for any profit-making activity or commercial gain

- You may freely distribute the URL identifying the publication in the public portal 


\begin{abstract}
1 ABSTRACT
2

3 Most of the current methods for controlling the formation rate of a key protein or enzyme in cell

4 factories rely on the manipulation of target genes within the pathway. In this article, we present a

5 novel synthetic system for post-translational regulation of protein levels, FENIX, which provides both

6 independent control of the steady-state protein level and inducible accumulation of target proteins.

7 The FENIX device is based on the constitutive, proteasome-dependent degradation of the target

8 polypeptide by tagging with a short synthetic, hybrid Nla/SsrA amino acid sequence in the C-terminal

9 domain. Protein production is triggered via addition of an orthogonal inducer (i.e. 3-methylbenzoate)

10 to the culture medium. The system was benchmarked in Escherichia coli by tagging two fluorescent

11 proteins (GFP and mCherry), and further exploited to completely uncouple poly(3-hydroxybutyrate)

12 (PHB) accumulation from bacterial growth. By tagging PhaA (3-ketoacyl-CoA thiolase, first step of the

13 route), a dynamic metabolic switch at the acetyl-coenzyme A node was established in such a way

14 that this metabolic precursor could be effectively re-directed into PHB formation upon activation of the

15 system. The engineered $E$. coli strain reached a very high specific rate of PHB accumulation $\left(0.4 \mathrm{~h}^{-1}\right)$

16 with a polymer content of ca. $72 \%(\mathrm{w} / \mathrm{w})$ in glucose cultures in a growth-independent mode. Thus,

17 FENIX enables dynamic control of metabolic fluxes in bacterial cell factories by establishing post-

18 translational synthetic switches in the pathway of interest.
\end{abstract}


One of the main challenges in contemporary metabolic engineering is to develop systems for

5 output ${ }^{1-2}$. The problem can be tackled by manipulating genes and proteins in cell factories at different

6 levels of regulation. Transcriptional and translational regulation mechanisms, for instance, have been

7 studied in great detail in many biotechnologically-relevant microorganisms, and several studies

8 describe synthetic circuits exploiting these cellular processes for bioproduction purposes ${ }^{3-6}$. More

9 recently, the adoption of CRISPR/Cas9-mediated technologies has opened up countless possibilities

10 for targeted regulation at the gene/genome level ${ }^{7-8}$. The conditional and dynamic control of protein

11 levels in vivo, in contrast, has received less attention thus far, and the majority of the currently

12 available tools designed to modulate protein activity target mRNAs and protein synthesis rates (e.g.

13 by using specific transcriptional repressors, RNA interference strategies, and riboregulators). Some

14 synthetic devices for the tunable control of protein synthesis and degradation have been developed

15 over the last few years ${ }^{9}$, e.g. systems triggered by small molecules ${ }^{10-12}$ or indirect degradation

16 processes $^{13-15}$. From a practical perspective, these strategies allow for a tight and accurate control of

17 metabolic pathways since the transcriptional or translational regulation of the gene(s) encoding the

18 target(s) are not altered.

Most approaches for bioproduction of added-value compounds usually rely on constitutive expression of the genes within the target pathway. Under these conditions, biosynthesis of the compound(s) of interest is simultaneous with bacterial growth. Growth-coupled production, however, severely limits product yield and productivity ${ }^{16-18}$. Biomass formation can consume up to $60 \%$ of the carbon source across different cultivation techniques. This situation is particularly relevant for products synthesized from precursors of central carbon metabolism that also serve as building blocks for biomass formation. Bacterial polyhydroxyalkanoates (PHAs), biodegradable polyesters with a broad range of biotechnological applications ${ }^{19-21}$, represent such an example, as many of them are synthesized from acetyl-coenzyme $\mathrm{A}(\mathrm{CoA})$ as main precursor ${ }^{22}$. Over the years, PHA production in recombinant Escherichia coli strains has mostly exploited growth-associated polymer accumulation ${ }^{23-24}$, which 
1 creates a competition for acetyl-CoA between biomass formation and PHA synthesis ${ }^{25}$ - potentially

2 leading to metabolic imbalances that hinder high levels of product accumulation. In this context, the

3 question at stake is whether the growth and production phases could be uncoupled by re-purposing

4 natural molecular mechanisms known to control protein integrity and functionality once the cognate

5 mRNAs have been translated.

6

7 Protein degradation in bacteria is mediated by several processes ${ }^{26}$. One of them is the so called 8 transfer-messenger RNA (tmRNA) system, based on special RNA molecules that function both as 9 tRNAs and mRNAs. tmRNAs form a ribonucleoprotein complex to recycle stalled ribosomes by nonstop mRNAs and tag incomplete nascent chains for degradation through the fusion of the SsrA 11 peptide ${ }^{27-28}$. In E. coli and related Gram-negative bacteria, this tag sequence is recognized by the 12 endogenous proteases ClpXP and ClpAP (both belonging to the proteasome complex), which rapidly 13 degrade the target protein. A separate proteolytic mechanism found in the prokaryotic world is the 14 processing of viral poly-proteins. The process is mediated by enzymes that target specific amino acid 15 sequences in otherwise very long polypeptide chains, thereby releasing functional individual proteins. 16 An archetypal example of poly-protein processing is based on the action of the so-called Nla 17 protease (nuclear inclusion protein $A)^{29-30}$. This enzyme was isolated from a virus of the Potyviridae 18 family (positive-sense single-stranded RNA genome) and it has the typical structural motifs of serine 19 proteases-although there is a cysteine residue instead of serine at the active site ${ }^{31-33}$. The Nla 20 protease has been used for the proteolytic removal of both affinity tags and fusion proteins from 21 recombinant target proteins, due to the stringent sequence specificity of the proteolytic cleavage (a 22 mere 13 amino acid sequence) $)^{34}$. Based on these properties, in this work we present FENIX (functional engineering of SsrA/Nla-based flux control), a novel tool that merges the two independent degradation systems mentioned above (i.e. tmRNAs and the Nla protease), for the sake of a rapid and convenient in vivo control of protein

27 activities in cell factories. To this end, a synthetic Nla/SsrA tag, which can be easily fused to the C28 terminal region of any given protein via a single cloning step in a standardized vector, was engineered to include sequences recognized by both the protease and the proteasome. Unlike other 
1 systems for post-transcriptional regulation, this strategy relies on the constitutive degradation of the

2 target followed by its conditional restoration. This system was instrumental to bring about an efficient decoupling of PHB accumulation from bacterial growth in recombinant $E$. coli strains by targeting a

4 key enzyme of the PHA biosynthesis machinery.

5

\section{RESULTS AND DISCUSSION}

Rationale of FENIX, a synthetic post-translational control system for pathway engineering. In

9 this work, a novel regulatory system at the post-translational level is presented that re-purposes the bacterial proteasome and combines its action with the specific protease Nla, the activity of which can

11 be externally controlled at the user's will. While typical control devices based on proteolysis eliminate 12 specific target proteins ${ }^{35-37}$, the FENIX system presented herein is based just on the opposite, i.e. the target is constitutively degraded by default by the endogenous proteasome until the conditional 14 activity of the Nla protease removes the degradation signals and enables the accumulation of the protein of interest (Fig. 1). A synthetic, hybrid tag sequence was designed, where the recognition sequence of the potyvirus Nla protease (GESNVWVHQADER) was fused to the SsrA target sequence (AANDENYALAA) recognized by the CIpXP and ClpAP components of the bacterial proteasome ${ }^{38}$. The synthetic Nla/SsrA tag (GESNVVVHQADER.AANDENYALAA) can be directly fused to the C-terminal domain of virtually any protein, rendering the corresponding polypeptide sensitive to rapid degradation by the proteasome system ${ }^{39}$ and abolishing protein accumulation and/or activity (Fig. 1a). In the presence of the Nla protease, in contrast, the proteolytic activity cleaves off the Nla/SsrA tag between the $Q$ and A residues of the tagged polypeptide, which will then release the SsrA target sequence from the C-terminus, thereby allowing for protein accumulation and/or enzyme activity (Fig. 1a).

In order to implement this scheme, a novel set of plasmids was constructed, based on the structure set by the Standard European Vector Architecture ${ }^{40-41}$, to facilitate the direct tagging of virtually any protein sequence with the synthetic Nla/SsrA tag (Fig. 1b; see details in Methods). The FENIX vectors (Table 1) enable simple exchange of the gene encoding a fluorescent protein by the gene of 
1 interest by digestion and ligation using the unique Nhel and BsrGl restriction sites. The resulting

2 pFENIX plasmid expresses a nia/ssrA-tagged version of the selected gene under the transcriptional

3 control of the constitutive $\mathrm{P}_{\text {teta }}$ promoter. An auxiliary plasmid, termed pS238. $\mathrm{Nla}$, was also

4 constructed for the regulatable expression of the gene encoding the Nla protease by placing the

5 cognate coding sequence under the transcriptional control of the XyIS/Pm expression system (Table

6 1), inducible upon addition of 3-methylbenzoate (3-mBz). With these plasmids at hand, we set out to

7 calibrate the FENIX system as indicated in the next section.

9 The FENIX system enables precise control of protein accumulation in recombinant $E$. coli strains. The first attempts at calibrating the FENIX system involved two fluorescent reporter proteins,

11 the commonly-used green fluorescent protein (GFP) and the red fluorescent protein mCherry. The 12 genes encoding these reporter proteins were individually fused to the synthetic hybrid nia/ssrA tag in 13 plasmids pFENIX'gfp and pFENIX·mCherry", respectively [Table 1; note that the asterisk symbol (*) 14 indicates the addition of the synthetic Nla/SsrA tag to the corresponding polypeptide]. Each plasmid 15 was separately transformed along with plasmid pS238-Nla into E. coli DH10B. When either GFP* or mCherry* are produced in $E$. coli, they will be rapidly degraded by the proteasome, i.e. no green or 17 red fluorescence would be observed under these conditions. Inspection of the plates in which the $E$. coli recombinants were streaked under blue light indicated that this was the case, as the colonies had no visually-detectable fluorescence (data not shown). In these strains, inducing the expression of nia from plasmid pS238-Nla would ultimately result in the removal of the SsrA tag, and the proteasome 21 would no longer be able to degrade the fluorescent proteins, which could thus be detected once they 22 accumulate in the cells at sufficient levels. To explore the kinetic properties of the FENIX system, these recombinant $E$. coli strains were grown in multi-well microtiter plates in LB medium with the 24 antibiotics and additives (3-mBz) indicated in Methods, and bacterial growth and fluorescence (GFP or mCherry) were recorded after $24 \mathrm{~h}$ of incubation at $37^{\circ} \mathrm{C}$ (Fig. 2).

27 The results of population-level fluorescence indicated that the qualitative behavior of the FENIX 28 system was reproducible irrespective of the fluorescent protein being tagged. When the tagged GFP* or mCherry* proteins were exposed to the action of the Nla protease, the levels of fluorescence 
1 attained after $24 \mathrm{~h}$ of cultivation were similar to those observed in the positive controls, in which the 2 genes encoding the native (i.e. non-tagged) GFP or mCherry proteins were constitutively expressed

3 from the $P_{\text {tetA }}$ promoter (Fig. 2). In the case of GFP*, the final fluorescence levels were ca. $70 \%$ of 4 those observed for GFP; for mCherry*, the fluorescence output was ca. $90 \%$ of that observed for the 5 non-tagged version of the protein. The FENIX system also exhibited remarkably low levels of either GFP* $^{*}$ or mCherry* fluorescence in the absence of 3-mBz, which indicates that the (potential) leaky

7 expression of nia does not significantly affect the output fluorescence (i.e. $<10 \%$ of the fluorescence 8 levels observed upon induction of the system in both cases)-thereby enabling tight control of protein 9 accumulation. Moreover, in order to explore the possible effects of the inducer of nia expression (3$\mathrm{mBz}$ ) on the behavior of the system, we also measured the specific fluorescence in cultures of $E$. coli

11 harboring only plasmids pFENIX·gfp* or pFENIX·mCherry* in the presence or the absence of 3-mBz.

12 As indicated in Fig. 2, the levels of specific fluorescence in either case were as low as the negative 13 control (i.e. no fluorescent protein), irrespective of the presence of 3-mBz. These quantitative results 14 were mirrored by the fluorescence observed in bacterial pellets of the recombinant cells harvested 15 from shake-flask cultures grown under the same conditions (Fig. 2, lower panel). Taken together, these results demonstrate that the FENIX system is functional in $E$. coli under the conditions tested, and that the proposed strategy can be established as a model for synthetic post-translational regulation. The next relevant question was to address the kinetic behavior of the system by means of flow cytometry.

The FENIX system enables a precise and concerted temporal switch of protein accumulation. The experiments described in the preceding section analyzed the behavior of the FENIX system at the whole-population level. To inspect fluorescence levels at the single-cell level, E. coli DH10B was transformed both with plasmid pS238.Nla and plasmid pFENIX·gfp* and the cultures were analyzed by flow cytometry (Fig. 3). In this case, the cells were grown in LB medium in shake-flask cultures under the same culture conditions used in the experiments carried out in microtiter-plate cultures, and samples were periodically taken to analyze the levels of GFP* fluorescence by flow cytometry. At the first data point, taken at $3 \mathrm{~h}$ post-induction of the system by the addition of 3-mBz at $1 \mathrm{mM}$, the induced (i.e. GFP*-positive) bacterial culture behaved as a single population (i.e. characterized by a 
1 single peak in the histogram plot of cell count versus GFP* fluorescence; Fig. 3a, first panel), clearly

2 distinguishable from the non-induced bacterial population (i.e. cultures grown in the absence of 3-

$3 \mathrm{mBz}$ ). This observation indicates that the operation of the FENIX system does not result in a mixture

4 of sub-populations of induced and non-induced cells. The level of GFP* fluorescence rapidly

5 increased after 5 and $8 \mathrm{~h}$ post-induction (Fig. 3a, second and third panel) and plateaued at $24 \mathrm{~h}$ (Fig.

$63 a$, fourth panel) at fluorescence values slightly below those observed in the positive control (i.e. E.

7 coli DH10B transformed with plasmid $p S 341 \mathrm{~T} \cdot g f p^{*}$, which constitutively expresses a GFP variant

8 displaying exactly the same amino acid sequence of the Nla/SsrA-tagged GFP after digestion by the

9 Nla protease; see Methods for details on the construction). Interestingly, the non-induced cultures

10 exhibited levels of GFP* fluorescence within the range of the strain used as a negative control (i.e. $E$.

11 coli DH10B transformed with plasmid pS238-Nla) throughout the whole cultivation period-thus

12 indicating a very low level of leakiness of the FENIX system in the absence of any inducer.

13

14 When the induction levels were calculated in this experiment (i.e. GFP* fluorescence in cells from

15 induced cultures as compared to those in the non-induced control experiments), a linear increase in

16 the fluorescence fold-change was observed over time (Fig. 3b). By the end of the experiment (i.e. 24

$17 \mathrm{~h}$ post-induction with 3-mBz), the GFP* fluorescence levels in cultures of $E$. coli DH10B transformed

18 both with plasmids pS238-Nla and pFENIX·gfp* was 24-fold higher than those observed in the non-

19 induced cultures of the same strain (and ca. 60-fold higher than those in cultures of E. coli DH10B

20 transformed only with plasmid pS238. $\mathrm{Nla}$, used as the negative control in these experiments). These

21 results confirm the versatility of the FENIX system to externally control the accumulation of a target

22 protein in a tightly regulated, and temporally coordinated fashion. Once the calibration of the system

23 was complete, the FENIX device was exploited for tackling a longstanding problem in metabolic

24 engineering of biopolymers as disclosed below.

25

26

27

28

29

\section{Establishing a FENIX-based metabolic switch for biopolymer accumulation in recombinant $E$.} coli strains. E. coli is a suitable host for engineering biopolymer biosynthesis as it lacks the machinery needed for PHA accumulation and degradation ${ }^{42}$, offering the flexibility to manipulate both native and heterologous pathways for biopolymer production ${ }^{43-44}$. PHAs are ubiquitous polymers that 
1 attract increasing industrial interest as renewable, biodegradable, biocompatible, and versatile 2 thermoplastics ${ }^{45}$. Poly(3-hydroxybutyrate) (PHB) is the structurally simplest and most widespread example of PHA in which the polymer is composed by C4 (i.e. 3-hydroxybutyrate) units. The archetypal PHB biosynthesis pathway of the Gram-negative bacterium Cupriavidus necator comprises three enzymes ${ }^{46}$ that use acetyl-CoA as the precursor and NADPH as the redox cofactor (Fig. 4a). PhaA, a 3-ketoacyl-CoA thiolase, condenses two acetyl-CoA moieties to yield 3acetoacetyl-CoA. This intermediate is the substrate for PhaB1, a NADPH-dependent 3-acetoacetylCoA reductase. In the final step, $(R)-(-)-3$-hydroxybutyryl-CoA is polymerized to PHB by the PhaC1 short-chain-length PHA synthase. Expression of the phaC1AB1 gene cluster from C. necator in $E$. coli results in glucose-dependent accumulation of PHB, and several examples of metabolic engineering of biopolymer accumulation have been published over the last few decades ${ }^{19-20}$. Yet, the spatio-temporal control of biopolymer accumulation in recombinant bacteria continues to prove challenging ${ }^{47}$ - particularly when attempting to balance the pathway at the level of gene expression ${ }^{48}$. On one hand, draining of acetyl-CoA away from central carbon metabolism interferes with bacterial growth if the PHB biosynthetic pathway is expressed during the active growth phase. On the other hand, acetyl-CoA is a hub metabolite in the cell, used as a precursor by a large number of metabolic pathways, and achieving precursor levels leading to high levels of PHB accumulation is inherently difficult considering the number of competing routes that also use acetyl-CoA. We hypothesized that the efficient uncoupling of bacterial growth and biopolymer accumulation could be an alternative for efficient PHB biosynthesis. Accordingly, the FENIX system was adapted to artificially control the level (and hence, the activity) of PhaA, the first committed step of the PHB biosynthesis pathway-and bottleneck of the entire route ${ }^{49}$ at the post-translational level in recombinant E. coli strains (Fig. $\mathbf{4 b}$ ). In order to tackle this challenge, phaA, the second gene in the phaC1AB1 gene cluster, was added with the synthetic, hybrid nia/ssrA tag fragment at the 3 '-end of the coding sequence (i.e. C-terminal domain of the protein) as indicated in Methods. The resulting engineered protein, $\mathrm{PhaA}^{*}$, is constitutively degraded by the bacterial proteasome unless the SsrA tag is removed from the polypeptide (by means of the Nla protease). On this background, the synthetic metabolic switch for controlled PHB accumulation based on the FENIX system was characterized as indicated in the next section. 
2 The PhaA activity can be tightly regulated by means of the FENIX system. E. coli BW25113, a

3 well characterized wild-type strain ${ }^{50}$, was transformed both with plasmids pS238.Nla and 4 pFENIX·PHA* (Table 1). Plasmid pFENIX·PHA* expresses the phaC1AB1 gene cluster of C. necator

5 from its own constitutive promoter, and contains a variant of phaA fused to the nia/ssrA-tag sequence

6 (Fig. 4b). Shake-flask cultures of this recombinant strain were carried out in LB medium containing

$730 \mathrm{~g} \mathrm{~L}^{-1}$ glucose, and growth, PHB accumulation, and in vitro PhaA activity were periodically 8 monitored over $24 \mathrm{~h}$ (Fig. 5). We first explored if the PhaA activity can be switched on by means of

9 the FENIX system. In non-induced cultures (i.e. without addition of 3-mBz), the levels of 3-ketoacyl10 CoA thiolase activity consistently remained below $2 \mu \mathrm{mol} \mathrm{min}{ }^{-1} \mathrm{mg}_{\text {protein }}{ }^{-1}$ throughout the cultivation 11 (Fig. 5a). This background thiolase activity was also detected in E. coli BW25113 transformed only 12 with plasmid pS238-Nla, and can be accounted for by the endogenous ketoacyl-CoA thiolases of $E$. 13 coli (e.g. AtoB and FadA $)^{51}$. In contrast, a quick and sharp increase in the in vitro PhaA activity was 14 detected when 3-mBz was added to the cultures at $1 \mathrm{mM}$, reaching a 30-fold higher level at $8 \mathrm{~h}$ post15 induction. By $24 \mathrm{~h}$ of cultivation, the PhaA activity in induced cultures had attained $6.1 \pm 0.7 \mu \mathrm{mol}$ $16 \mathrm{~min}^{-1} \mathrm{mg}_{\text {protein }}{ }^{-1}$. In a parallel experiment, E. coli BW25113/pS238. Nla was transformed either with 17 plasmids pAeT41 or pS341.PHA, which constitutively express the native phaC1AB1 gene cluster of 18 C. necator (in the latter case, in the same vector backbone used for pFENIX plasmids, i.e. pSEVA341). The in vitro PhaA activity was measured in 24-h cultures of these recombinant strains under the same growth conditions indicated above, in the absence of presence of 3-mBz (Fig. 5b). E. coli BW25113/pS238-Nla transformed either with plasmids pAeT41 or pS341·PHA had similarly high levels of PhaA activity irrespective of the presence of 3-mBz. In contrast, a clear difference in the thiolase activity was detected in E. coli BW25113 transformed both with plasmids pS238.Nla and pFENIX.PHA* In non-induced cultures, the enzymatic activity remained at levels $<1 \mu \mathrm{mol} \mathrm{min}^{-1}$

$25 \mathrm{mg}_{\text {protein }}{ }^{-1}$ even after $24 \mathrm{~h}$ of cultivation, but the addition of 3-mBz triggered an 8-fold increase in PhaA 26 activity. Moreover, the activity in the induced cultures carrying the $\mathrm{PhaA}^{*}$ variant reached the highest 27 levels among all experimental strains and conditions. The tighter control of protein accumulation 28 afforded by the FENIX system thus contributed to 1.6-fold higher activity levels of the tagged enzyme 29 as compared to the native PhaA enzyme. Since the levels of gene expression in the plasmids tested 
1 (as well as the copy number of the cognate replicons) are expected to be similar, it is likely that the

2 high PhaA activity is related to the rapid accumulation of the thiolase enzyme, draining acetyl-CoA

3 into the PHB biosynthesis pathway-a result closely matched by polymer accumulation as indicated

4 below.

5

6 The levels of PHB accumulation were also inspected in these cultures by means of flow cytometry

7 and gas chromatography as indicated in Methods. The content of PHB in the bacterial biomass

8 closely mirrored the levels of PhaA activity in all recombinant strains (Fig. 5c). Again, 3-mBz-induced

9 cultures of the strain carrying the Nla/SsrA-tagged variant of PhaA exhibited the highest polymer 10 content on a cell dry weight (CDW) basis among all strains tested [56.2\% $\pm 6.1 \%(\mathrm{w} / \mathrm{w}), 7$-fold higher

11 than that in non-induced cultures]. The final PHB content in strains carrying either plasmid pAeT41 or $12 \mathrm{pS} 341 \cdot \mathrm{PHA}$ was similar irrespective of the addition of $3-\mathrm{mBz}[<45 \%(\mathrm{w} / \mathrm{w})]$, whereas the cells 13 carrying pFENIX·PHA* had a negligible level of polymer accumulation in the absence of the inducer $14[<8 \%(w / w)]$. Importantly, all the strains grew at similar levels (with a final biomass density of ca. 5 $15 g_{C D W} L^{-1}$ at $24 \mathrm{~h}$ ), indicating that the differences observed in PHB accumulation across the strains can 16 be attributed to the dynamics of PhaA* activity brought about by the FENIX system and not to any 17 effect on bacterial growth or gene expression level.

\section{The FENIX system enables efficient decoupling of PHB biosynthesis and bacterial growth and} leads to high rates of biopolymer accumulation. In order to gain further insights into the dynamics of $\mathrm{PHB}$ accumulation in recombinant $E$. coli strains in shake-flask cultures, a thorough physiological characterization was carried out in M9 minimal medium containing $30 \mathrm{~g} \mathrm{~L}^{-1}$ glucose as the sole carbon source (Fig. 6). To this end, bacterial growth and PHB accumulation were closely monitored over $24 \mathrm{~h}$ in batch cultures of $E$. coli BW25113/pS238-Nla carrying either plasmid pS341.PHA (native PhaA) or pFENIX'PHA* (Nla/SsrA-tagged PhaA). The growth of the two strains was comparable, and the final biomass density plateaued at ca. $3.5 \mathrm{gcDW} \mathrm{L}^{-1}$ (Fig. 6a). The trajectory of PHB accumulation, in contrast, differed between the two strains (Fig. 6b). In E. coli BW25113/pS238. Nla carrying pS341.PHA, the amount of PHB increased exponentially throughout the cultivation period (i.e. closely resembling biomass formation), whereas in the strain carrying the $\mathrm{PhaA}^{*}$ variant the accumulation of 
1 PHB was clearly dissociated from bacterial growth, with biopolymer levels consistently $<5 \%(\mathrm{w} / \mathrm{w})$

2 during the first $8 \mathrm{~h}$ of cultivation. Once PHB accumulation was triggered, it rapidly increased

3 exponentially. Similarly to the observation made in LB cultures, the strain carrying the $\mathrm{Nla} / \mathrm{SsrA}-$

4 tagged version of PhaA attained a higher PHB content in these glucose cultures $[72.4 \% \pm 1.8 \%$

5 (w/w), 1.3-fold higher than that in the strain expressing the native phaC1AB1 gene cluster, $P<0.05$;

6 Fig. 6b].

7

8 Next, we assessed the specific rate of bacterial growth and biopolymer accumulation (Fig. 6c). The

9 specific growth rate $(\mu)$, as inferred from the growth curves, was not significantly different between

10 the two recombinant $E$. coli strains (ca. $0.3 \mathrm{~h}^{-1}$ ). However, the temporal separation of PHB

11 accumulation from bacterial growth in the strain carrying the $\mathrm{PhaA}^{*}$ variant resulted a 2 -fold higher

12 specific rate of PHB accumulation $\left(r_{\mathrm{PHB}}\right)$. Under these experimental conditions, $r_{\mathrm{PHB}}=0.41 \pm 0.02 \mathrm{~h}^{-1}$,

13 the highest reported in the literature for recombinant $E$. coli strains. The growth decoupling effect was

14 also evidenced when cells were sampled from these cultures, stained with the lipophilic Nile Red dye,

15 and observed under the fluorescence microscope (Fig. 6d). Upon induction of the FENIX system, the

16 rapid accumulation of PHB in the recombinant cells could be clearly detected as the polymer

17 granules started to fill the bacterial cytoplasm. Taken together, these results suggest that the FENIX

18 device can be used as a metabolic switch to enhance PHB production by controlling fluxes around

19 the acetyl-CoA metabolic node-a possibility that was explored in detail as explained below.

21 Enhanced PHB accumulation mediated by $\mathrm{PhaA}^{*}$ stems from flux re-wiring around the acetyl-

22 CoA node. As indicated previously, acetyl-CoA is a metabolic hub in the cell. In the recombinant $E$. 23 coli strains described in this work, a major competition occurs at this node between the PHB 24 biosynthesis pathway and other endogenous metabolic routes. Apart from the core cell functions that 25 use acetyl-CoA as building-block (e.g. de novo fatty acid synthesis), in the presence of excess 26 glucose, E. coli synthesizes (and excretes) acetate from acetyl-CoA through a two-step route 27 catalyzed by Pta (phosphotransacetylase) and AckA (acetate kinase) ${ }^{52}$ (Fig. 7a). Taking advantage 28 of this biochemical feature, the specific rate of acetate formation and the content of acetyl-CoA were 29 adopted as a proxy to gauge how the FENIX device could re-direct this metabolic precursor into a 
1 target pathway. A lower specific rate of acetate formation was detected in glucose cultures of all $E$.

2 coli strains expressing the PHB biosynthesis pathway as compared to the control strain, transformed

3 with the empty pSEVA341 vector (Fig. 7b) - consistent with a higher flux of acetyl-CoA funneled into

4 PHB formation. However, E. coli BW25113/pS238-Nla transformed with plasmid pFENIX·PHA* had

5 the lowest rate of acetate synthesis along all the strains tested $\left(0.9 \pm 0.1 \mathrm{mmol} \mathrm{g}_{\mathrm{cDw}}{ }^{-1} \mathrm{~h}^{-1} ; 70 \%\right.$ lower

6 than that of the control strain). Interestingly, when the specific rates of glucose consumption were

7 also determined in these cultures, no major differences were observed among all the strains (with $q_{s}$

8 values around $\left.7-8 \mathrm{mmol}_{\mathrm{CDW}^{-1}} \mathrm{~h}^{-1}\right)$, indicating that the differences in acetate formation or PHB

9 accumulation are linked to a re-routing of acetyl-CoA rather than to significant changes in the overall cell physiology.

12 The intracellular acetyl-CoA content qualitatively followed the same trend as the specific rates of 13 acetate formation, although the values obtained for this parameter were comparable among the 14 control strain and recombinant E. coli expressing the native phaC1AB1 gene cluster (Fig. 7c). Again, 15 the tight control of the PHB biosynthesis pathway at the level of PhaA afforded by the FENIX system 16 was reflected in the lowest content of acetyl-CoA among all strains tested $\left(0.23 \pm 0.05 \mathrm{nmol}_{\mathrm{CDw}}^{-}\right.$ 17 1)-suggesting an efficient re-routing of this metabolic precursor into PHB accumulation rather than 18 into other metabolic sinks of acetyl-CoA. These results certify that the FENIX system could be used 19 to establish an orthogonal control in key metabolic nodes of the biochemical network, acting as an 20 efficient switch to re-route fluxes around such nodes towards the biosynthesis of a product of interest.

Re-programming microorganisms to modify existing cell functions and to bestow cell factories with new-to-Nature tasks have largely relied on the implementation of specialized molecular biology 26 tools - which, for the most part, tackle the issue at the genetic level of regulation. More recently, 27 novel approaches for pathway engineering were designed to also encompass the dynamic regulation 28 of protein levels. Most of these examples of this type of approaches, however, rely on the controlled 29 degradation of a target polypeptide to create a conditional phenotypic knock-outs ${ }^{10,9}$. To the best of 
1 our knowledge, the FENIX device described in this work exploits a hitherto unexplored feature, 2 namely, the constitutive degradation of a target protein within a pathway, the production of which can

3 be triggered at the user's will by addition of a cheap inducer (i.e. 3-mBz) to the culture medium. 4 Besides the metabolic engineering application discussed in the present study (i.e. biopolymer 5 accumulation in recombinant $E$. coli strains by targeting PhaA, the first enzymatic activity of the 6 pathway), the FENIX system affords more complex pathway engineering approaches in which the

7 formation of multiple proteins within different domains of the metabolic network can be externally 8 controlled. We have selected the intracellular accumulation of PHB as a case study for the 9 manipulations described in this work, but the system could be likewise adopted to increase the biosynthesis of extracellular products (especially toxic or highly-reactive molecules such as complex

11 alcohols or ketones-the production of which would be difficult to tightly control at the gene 12 expression level${ }^{53-54}$ ) and to reduce metabolic burden due to heterologous protein production². The 13 system could be also used for physiological studies based on gain-of-function in single or multiple 14 metabolic nodes in a biochemical network in vivo ${ }^{55}$. Our results indicate that the addition of the SsrA 15 and Nla tags into a polypeptide does not affect its folding or function significantly-providing evidence 16 that the system can be adapted to other targets beyond the proteins described herein. The tight post17 translational regulation of this system enables product titers that would be difficult to achieve by 18 merely manipulating expression of the cognate genes ${ }^{56}$ at the transcriptional level, as most inducible 19 systems are typically leaky ${ }^{57}$ and the induction kinetics slow ${ }^{58-59}$. Moreover, and considering the 20 dynamic response of FENIX-tagged proteins accumulation, the system would also allow for the 21 expression of highly toxic proteins or enzymes, which would otherwise result in. These scenarios are 22 currently under exploration in our laboratory and may lead to the development of better strategies to 23 manipulate central and peripheral pathways to enhance the production of biochemicals and other 24 molecules of industrial interest.

25

28 Bacterial strains and cultivation conditions. The E. coli strains and plasmids used in this study are 29 listed in Table 1. E. coli was grown at $37^{\circ} \mathrm{C}$ in LB medium ${ }^{60}$ or in $\mathrm{M} 9$ minimal medium ${ }^{61}$ added with 
1 glucose $\left(30 \mathrm{~g} \mathrm{~L}^{-1}\right)$ as the sole carbon source. For solidified culture media, 1.5\% (w/v) agar was used.

2 Shake-flask cultivations were routinely carried out in an air incubator with orbital shaking at $200 \mathrm{rpm}$. Aerobic cultures were set by using a 1:10 culture medium-to-flask volume ratio. Antibiotics were

4 added to the cultures where appropriate at the following final concentrations: ampicillin (Ap, $150 \mathrm{mg}$

$\left.5 \mathrm{~L}^{-1}\right)$, chloramphenicol $\left(\mathrm{Cm}, 30 \mathrm{mg} \mathrm{L}^{-1}\right)$, and kanamycin $\left(\mathrm{Km}, 50 \mathrm{mg} \mathrm{L}^{-1}\right)$.

General molecular biology techniques. Recombinant DNA techniques were carried out by following well established methods ${ }^{62}$. Plasmid DNA was prepared from recombinant $E$. coli with a High-Pure plasmid isolation kit (Roche Applied Science). DNA fragments were purified from agarose gels with the Gene-Clean Turbo kit (Q-BIOgene). Oligonucleotides were purchased from SigmaAldrich Co. The identity of all cloned inserts and DNA fragments was confirmed by DNA sequencing through an ABI Prism 377 automated DNA sequencer (Applied Biosystems Inc.). Transformation of E. coli cells with plasmids was routinely carried out by means of the $\mathrm{RbCl}$ method or by electroporation ${ }^{62}$ (Gene Pulser, Bio-Rad).

\section{Design and construction of pFENIX plasmids carrying proteolizable versions of GFP and} mCherry. The general strategy for the assembly of pFENIX plasmids is indicated in Fig. 1b. In all the constructs described in this article, the asterisk symbol $\left({ }^{*}\right)$ indicates that the corresponding gene has been added with a synthetic nia/ssrA tag. The starting point was the creation of plasmids pFENIX·gfp ${ }^{*}$ and pFENIX'mCherry ${ }^{*}$ as follows: the nia/ssrA tag was firstly assembled using the synthetic oligonucleotides 5'-nia/ssrA·BsrGI (5'-GAG CTG TAC AAG GGT GAA AGC AAC GTG gtg gtg cat cag gcg gat gaa cgc gca gca aac gac gaa aac-3'; an engineered BsrGI site, not present in SEVA vectors ${ }^{40}$, is underlined) and 3'-nia/ssrA HindIII (5'-CCC AAG CTT TTA AGC TGC TAA AGC GTA gtt ttc gtc gtt tgc tgc gcg ttc atc cgc ctg atg cac cac-3'; an engineered Hindlll site is underlined). The 42-bp long DNA sequence indicated in lowercase letters in these two oligonucleotides was used as an overlapping extension for sewing PCR, and the whole 89-bp long DNA fragment spanning the synthetic nia/ssrA tag was amplified with Pfu DNA polymerase (Promega) as per the manufacturer's instructions. Plasmid pS341T was constructed by cloning the $P_{\text {tetA }}$ promoter (which, in the absence of the TetR negative regulator ${ }^{63}$, acts as a medium-strength constitutive promoter in Gram-negative 
1 bacteria ${ }^{64}$ ) between the Pacl and EcoRI restriction targets of vector PSEVA341, and a Nhel restriction

2 target, not present in SEVA vectors, was added to the construct to facilitate further cloning. Plasmid

$3 \quad \mathrm{pS} 341 \mathrm{~T} \cdot \mathrm{mCherry}$ was constructed by placing the gene encoding the red fluorescent protein mCherry

4 under control of the $P_{\text {tetA }}$ promoter as a Xhol/Hindlll fragment obtained from vector pSEVA237R, and

5 a BsrGl restriction target was added upstream the mCherry coding sequence by PCR. The resulting $6 \mathrm{pS} 341 \mathrm{~T} \cdot \mathrm{mCherry}$ plasmid was further engineered to include the synthetic nia/ssrA tag by means of

7 sewing PCR. The tag was directly cloned as a BsrGI/Hindlll fragment downstream the mCherry gene, 8 thus giving rise to pFENIX·mCherry ${ }^{*}$ (Table 1$)$. The same procedure was repeated with the gene 9 encoding GFP, yielding pFENIX·gfp* (Table 1). Both plasmids were used to calibrate the FENIX system, and they allow for the easy construction of a proteolizable version of virtually any protein by a

11 direct cloning step of the corresponding gene of interest into the Nhel and BsrGl restriction sites that 12 flank the fluorescent protein coding sequence. Moreover, as the pFENIX plasmids described here 13 follow the formatting rules of the SEVA collection ${ }^{40}$, other parts (e.g. origins of replication, inducible 14 and constitutive promoters, antibiotic resistances, etc.) could be easily incorporated as needed.

Two expression vectors were also constructed as positive controls of the FENIX system. In order to 17 stablish a direct comparison between the fluorescence originated by the engineered GFP* or mCherry* fluorescent proteins after proteolysis, we designed and created a version of these two proteins that have the same amino acid sequence as the proteolizable variants after digestion by the Nla protease. Plasmid pS341T.mCherry ${ }^{*}$, encoding such an engineered mCherry protein, was constructed by amplifying the mCherry gene plus the short sequence of the nia target that remains after protease digestion using oligonucleotides 5'-mCherry•Nhel (5'-CAC AGG AGG GCT AGC ATG GTG AG-3'; an engineered Nhel site is underlined) and 3'-mCherry·HindIII (5'-GGG AAG CTT TTA CTG ATG CAC CAC CAC GTT GCT TTC-3'; an engineered Hindlll site is underlined) by using plasmid pFENIX·mCherry* as the template. The resulting amplicon, which spans the sequence encoding the mCherry protein after proteolysis, was restricted with the enzymes indicated and cloned into the Nhel/Hindlll-digested pS341T vector, thereby obtaining plasmid pS341T·mCherry* (Table 1). 


\section{Construction of plasmid pFENIX.PHA* for post-translational control of PHB accumulation in} recombinant $\boldsymbol{E}$. coli strains. Since phaA lies in the middle of the pha gene cluster of $C$. necator, the strategy used for tagging this gene was slightly different as the one described above for single-gene targets. In this case, the synthetic nia/ssrA tag was firstly added to phaA by overlapping PCR. Two individual DNA fragments upstream and downstream with respect to the STOP codon of phaA were amplified by PCR using oligonucleotides (i) 5'-phaA-Bg/ll (5'-CAC GCG GCA AGA TCT CGC AGA CC-3'; an engineered Bglll site is underlined) and 3'-phaA.nia (5'- cgt cgt ttg ctg cgc gtt cat ccg cct gat gca cca cca cgt tgc ttt cac cTT TGC GCT CGA CTG CCA GCG C-3') for the upstream fragment (2,462 bp) and (ii) 5'-phaA·nia (5'-gca tca ggc gga tga acg cgc agc aaa cga cga aaa cta cgc ttt agc agc tTA AGG AAG GGG TTT TCC GGG GC-3') and 3'-phaA·EcoRI (5'-GAC CAT GAT TAC GAA TTC TTC TGA ATC CAT G-3'; an engineered EcoRI site is underlined) for the downstream fragment $(1,398 \mathrm{bp})$. Both amplicons were used to construct a DNA fragment spanning phaA and the synthetic nia/ssrA tag by sewing PCR using the overlapping sequences in the oligonucleotides 5'-phaA nia and 3'-phaA-nia (indicated in lowercase letters). This DNA fragment was cloned into the Bglll/EcoRIdigested plasmid pAET41, obtaining plasmid pAeT41.PHA*, in which the native phaA sequence has been exchanged by the nia/ssrA tagged version of the same gene. Plasmid pAeT41·PHA* was then used as the template for a PCR amplification of the engineered pha gene cluster by using oligonucleotides 5'-PHA·BamHI (5'-AGA GGA TCC GGA CTC AAA TGT CTC GGA ATC GCT G-3'; an engineered BamHI site is underlined) and 3'-PHA.EcoRI (5'-GCG AAT TCC ACC GCA ATA CGC GGG CGC CAG-3'; an engineered EcoRI site is underlined). The resulting amplicon (4,292 bp) was digested with BamHI and EcoRI and cloned into the same restriction sites of vector pSEVA341, resulting in plasmid pFENIX.PHA*. To test PHB accumulation using a comparable vector system, plasmid pS341.PHA was constructed as follows. The native pha gene cluster was amplified by PCR from plasmid pAeT41 as the template using oligonucleotides 5'-PHA.BamHI and 3'-PHA.EcoRI. The resulting DNA fragment (4,220 bp) was digested with BamHI and EcoRI and cloned into the same restriction sites of vector pSEVA341, resulting in plasmid pS341.PHA. Note that these vectors contain compatible replicons (derived from the origin of replication of plasmid pMB1) and display similar copy numbers in E. colito. E. coli BW25113 was transformed with plasmid pS238.Nla and either pS341.PHA or pFENIX·PHA*, and tested for PHB accumulation as indicated below. 
2 Flow cytometry evaluation of the FENIX system. Single-cell fluorescence was analyzed with a MACSQuant $^{T M}$ VYB cytometer (Miltenyi Biotec $\mathrm{GmbH}$ ). GFP was excited at $488 \mathrm{~nm}$, and the

4 fluorescence signal was recovered with a $525 / 40 \mathrm{~nm}$ band pass filter. Cells were harvested at

5 different time points as indicated in the text, and at least 15,000 events were analyzed for every

6 aliquot. The GFP signal was quantified under the experimental conditions tested by firstly gating the

7 cells in a side scatter against forward scatter plot, and then the GFP-associated fluorescence was

8 recorded in the FL1 channel $(515-545 \mathrm{~nm})$. Data processing was performed using the FlowJo ${ }^{\mathrm{TM}}$

9 software as described elsewhere ${ }^{65}$.

11 In vitro quantification of the PhaA activity. Cell-free extracts were obtained from bacteria 12 harvested by centrifugation $\left(4,000 \times \mathrm{g}\right.$ at $4^{\circ} \mathrm{C}$ for $\left.10 \mathrm{~min}\right)$. Cell pellets were resuspended in $1 \mathrm{~mL}$ of a 13 lysis buffer containing $10 \mathrm{mM}$ Tris $\cdot \mathrm{HCl}(\mathrm{pH}=8.1), 1 \mathrm{mM}$ EDTA, $10 \mathrm{mM} \beta$-mercaptoethanol, 20\% (v/v) 14 glycerol, and $0.2 \mathrm{mM}$ phenylmethylsulphonylfluoride, and lysed as described elsewhere ${ }^{66}$. The lysate 15 was clarified by centrifugation $\left(4^{\circ} \mathrm{C}, 10 \mathrm{~min}\right.$ at $\left.8,000 \times \mathrm{g}\right)$ and the resulting supernatant was used for enzyme assays. The total protein concentration was assessed by means of the Bradford method with 17 a kit from BioRad Laboratories, Inc. (USA), and crystalline bovine serum albumin as standard. In vitro 18 quantification of the specific 3-ketoacyl-CoA thiolase activity in the thiolysis direction was conducted 19 according to Palmer et al. ${ }^{67}$ and Slater et al. ${ }^{46}$, with the following modifications. The assay mixture (1 $\mathrm{mL})$ contained $62.4 \mathrm{mM}$ Tris $\cdot \mathrm{HCl}(\mathrm{pH}=8.1), 50 \mathrm{mM} \mathrm{MgCl}, 62.5 \mu \mathrm{M} \mathrm{CoA}$, and $62.5 \mu \mathrm{M}$ acetoacetyl-

21 CoA. The reaction was initiated by addition of cell-free extract, and the disappearance of acetoacetyl22 CoA was measured over time at $30^{\circ} \mathrm{C}$ (using $\varepsilon_{304}=16.9 \times 10^{3} \mathrm{M}^{-1} \mathrm{~cm}^{-1}$ as the extinction coefficient for 23 3-acetoacetyl-CoA). The actual acetoacetyl-CoA was routinely quantified prior to the assay in a buffer 24 containing $62.4 \mathrm{mM}$ Tris. $\mathrm{HCl}(\mathrm{pH}=8.1)$ and $50 \mathrm{mM} \mathrm{MgCl}_{2}$. One enzyme unit is defined as the amount of enzyme catalyzing the conversion of $1 \mu \mathrm{mol}$ of substrate per min at $30^{\circ} \mathrm{C}$.

27 PHB quantification. The intracellular polymer content in $E$. coli was quantitatively assessed by flow 28 cytometry by using a slight modification of the protocol of Tyo et al. ${ }^{68}$ and Martínez-García et al. ${ }^{69}$ 29 Cultures were promptly cooled to $4^{\circ} \mathrm{C}$ by placing them in an ice bath for $15 \mathrm{~min}$. Cells were harvested 
1 by centrifugation $\left(5 \mathrm{~min}, 5,000 \times \mathrm{g}, 4^{\circ} \mathrm{C}\right.$ ), resuspended to an $\mathrm{OD}_{600}$ of 0.4 in cold TES buffer [10 $\mathrm{mM}$ 2 Tris $\mathrm{HCl}(\mathrm{pH}=7.5), 2.5 \mathrm{mM}$ EDTA, and 10\% (w/v) sucrose], and incubated on ice for $15 \mathrm{~min}$.

3 Bacteria were recovered by centrifugation as explained above, and resuspended in the same volume 4 of cold $1 \mathrm{mM} \mathrm{MgCl}_{2}$. A $1-\mathrm{ml}$ aliquot of this bacterial suspension was added with $3 \mu \mathrm{L}$ of a $1 \mathrm{mg} \mathrm{mL}^{-1}$

5 Nile Red [9-diethylamino-5H-benzo( $\alpha$ )phenoxazine-5-one] solution in DMSO and incubated in the dark at $4^{\circ} \mathrm{C}$ for $30 \mathrm{~min}$. Flow cytometry was carried out in a MACSQuant ${ }^{\mathrm{TM}}$ VYB cytometer (Miltenyi

7 Biotec $\mathrm{GmbH}$ ). Cells were excited at $488 \mathrm{~nm}$ with a diode-pumped solid-state laser, and the Nile Red

8 fluorescence at $585 \mathrm{~nm}$ was detected with a $610 \mathrm{~nm}$ long band-pass filter. The analysis was done on 9 at least 50,000 cells and the results were analyzed with the built-in MACSQuantify ${ }^{\top M}$ software 2.5 (Miltenyi Biotec). The geometric mean of fluorescence in each sample was correlated to PHB content (expressed as a percentage) through a calibration curve. PHB accumulation was double-checked in selected samples by acid-catalyzed methanolysis of freeze-dried biomass and detection of the resulting methyl-esters of 3-hydroxybutyric acid by gas chromatography ${ }^{70-71}$. The specific rate of PHB accumulation $\left(r_{\mathrm{PHB}}\right)$ was analytically calculated during exponential polymer production, assessed in a semi-logarithmic plot of PHB content $\left(\mathrm{g}_{\mathrm{PHB}} / \mathrm{g}_{\mathrm{CDW}}\right)$ versus time (in $\mathrm{h}$ ) as indicated by van Wegen et al. ${ }^{49}$

For microscopic visualization of PHB accumulation ${ }^{72}$, cells harvested from shake-flask cultures were washed once with cold TES buffer, re-suspended in $1 \mathrm{~mL}$ of the same buffer to an $\mathrm{OD}_{600}$ of 0.4 , and stained with Nile Red as indicated for the flow cytometry experiments. Aliquots of the treated cell suspension were washed once with TES buffer, immediately lay in a microscope slide, and covered with a glass cover slip (to protect the stained cells from immersion oil). Images were obtained using an Axio Imager Z2 microscope (Carl Zeiss), equipped with the scanning platform Metafer 4 and 23 CoolCube 1 camera (MetaSystems) under a 1,000× magnification. Under these conditions, PHB 24 granules stained with Nile Red fluoresced bright orange, with individual granules often visible within 25 the cells.

Other analytical techniques. Residual glucose and acetate concentrations were determined in culture supernatants using enzymatic kits (R-Biopharm AG), essentially as per the manufacturer's instructions. Control mock assays were made by spiking M9 minimal medium with different amounts 
1 of the metabolite under examination. Metabolite yields and kinetic culture parameters were

2 analytically calculated from the raw growth data as described elsewhere ${ }^{66}$. The intracellular content of

3 acetyl-CoA was determined in samples taken during exponential bacterial growth by liquid

4 chromatography coupled to mass spectrometry as indicated by Pflüger-Grau et al. ${ }^{73}$

5

6 Statistical analysis. All reported experiments were independently repeated at least three times (as

7 indicated in the figure legends), and mean values of the corresponding parameter and standard

8 deviation is presented. The significance of differences when comparing results was evaluated by

9 means of the Student's $t$ test.

11 COMPETING INTERESTS

12 The authors declare that there are no competing interests.

\section{AUTHORS' CONTRIBUTIONS}

15 G.D.R. and P.I.N. carried out the genetic manipulations, quantitative physiology experiments, and in 16 vitro enzyme assays. G.D.R., V.D.L., and P.I.N. conceived the whole study, designed the 17 experiments, contributed to the discussion of the research and interpretation of the data, and wrote 18 the article.

\section{ACKNOWLEDGMENTS}

21 The authors are indebted to B. Calles (CNB-CSIC, Spain), M. H. Nørholm (Technical University of

22 Denmark, Denmark), and A. Sinskey (Massachusetts Institute of Technology, USA) for helpful 23 discussions and for sharing research materials. This study was supported by The Novo Nordisk 24 Foundation (Grant NNF10CC1016517) and the Danish Council for Independent Research (SWEET, 25 DFF-Research Project 8021-00039B) to P.I.N. This study was also supported by the HELIOS Project 26 of the Spanish Ministry of Economy and Competitiveness BIO2015-66960-C3-2-R 27 (MINECO/FEDER), and the ARISYS (ERC-2012-ADG-322797), EmPowerPutida (EUH202028 BIOTEC-2014-2015-6335536), and MADONNA (H2020-FET-OPEN-RIA-2017-1-766975) contracts of 29 the European Union to V.D.L. 
4 (1) Avcilar-Kucukgoze, I., and Ignatova, Z. (2017) Rewiring host activities for synthetic circuit

(2) Wu, G., Yan, Q., Jones, J. A., Tang, Y. J., Fong, S. S., and Koffas, M. A. G. (2016) Metabolic burden: cornerstones in synthetic biology and metabolic engineering applications. Trends Biotechnol. 34 (8), 652-664.

(3) Guzmán, L. M., Belin, D., Carson, M. J., and Beckwith, J. (1995) Tight regulation, modulation, and high-level expression by vectors containing the arabinose $P_{\mathrm{BAD}}$ promoter. $\mathrm{J}$. Bacteriol. 177 (14), 4121-4130.

(4) Isaacs, F. J., Dwyer, D. J., Ding, C., Pervouchine, D. D., Cantor, C. R., and Collins, J. J. (2004) Engineered riboregulators enable post-transcriptional control of gene expression. Nat. Biotechnol. 22 (7), 841-847.

(5) Lou, C., Stanton, B., Chen, Y. J., Munsky, B., and Voigt, C. A. (2012) Ribozyme-based insulator parts buffer synthetic circuits from genetic context. Nat. Biotechnol. 30 (11), 11371142.

(6) Lutz, R., and Bujard, H. (1997) Independent and tight regulation of transcriptional units in Escherichia coli via the LacR/O, the TetR/O and AraC/11-12 regulatory elements. Nucleic Acids Res. 25 (6), 1203-1210.

(7) Jakočiūnas, T., Jensen, M. K., and Keasling, J. D. (2017) System-level perturbations of cell metabolism using CRISPR/Cas9. Curr. Opin. Biotechnol. 46, 134-140.

(8) Shabestary, K., Anfelt, J., Ljungqvist, E., Jahn, M., Yao, L., and Hudson, E. P. (2018) Targeted repression of essential genes to arrest growth and increase carbon partitioning and biofuel titers in cyanobacteria. ACS Synth. Biol. 7 (7), 1669-1675.

(9) Tan, S. Z., and Prather, K. L. (2017) Dynamic pathway regulation: Recent advances and methods of construction. Curr. Opin. Chem. Biol. 41, 28-35. 
1 (10) Bonger, K. M., Chen, L. C., Liu, C. W., and Wandless, T. J. (2011) Small-molecule displacement of a cryptic degron causes conditional protein degradation. Nat. Chem. Biol. 7 (8), 531-537.

(11) Janssen, B. D., and Hayes, C. S. (2012) The tmRNA ribosome-rescue system. Adv. Protein Chem. Struct. Biol. 86, 151-191.

(12) Neklesa, T. K., Tae, H. S., Schneekloth, A. R., Stulberg, M. J., Corson, T. W., Sundberg, T. B., Raina, K., Holley, S. A., and Crews, C. M. (2011) Small-molecule hydrophobic tagginginduced degradation of HaloTag fusion proteins. Nat. Chem. Biol. 7 (8), 538-543.

(13) Cameron, D. E., and Collins, J. J. (2014) Tunable protein degradation in bacteria. Nat. Biotechnol. 32 (12), 1276-1281.

(14) Martínez, V., Lauritsen, I., Hobel, T., Li, S., Nielsen, A. T., and Nørholm, M. H. H. (2017) CRISPR/Cas9-based genome editing for simultaneous interference with gene expression and protein stability. Nucleic Acids Res. 45 (20), e171.

(15) Stein, V., Nabi, M., and Alexandrov, K. (2017) Ultrasensitive scaffold-dependent protease sensors with large dynamic range. ACS Synth. Biol. 6 (7), 1337-1342.

(16) Bothfeld, W., Kapov, G., and Tyo, K. E. J. (2017) A glucose-sensing toggle switch for autonomous, high productivity genetic control. ACS Synth. Biol. 6 (7), 1296-1304.

(17) Li, S., Jendresen, C. B., Grünberger, A., Ronda, C., Jensen, S. I., Noack, S., and Nielsen, A. T. (2016) Enhanced protein and biochemical production using CRISPRi-based growth switches. Metab. Eng. 38, 274-284.

(18) Volke, D. C., and Nikel, P. I. (2018) Getting bacteria in shape: Synthetic morphology approaches for the design of efficient microbial cell factories. Adv. Biosyst. In press, 1800111.

(19) Gomez, J. G. C., Méndez, B. S., Nikel, P. I., Pettinari, M. J., Prieto, M. A., and Silva, L. F. (2012) Making green polymers even greener: towards sustainable production of polyhydroxyalkanoates from agroindustrial by-products. In Advances in Applied Biotechnology, Petre, M., Ed. InTech: Rijeka, Croatia, pp 41-62.

(20) López, N. I., Pettinari, M. J., Nikel, P. I., and Méndez, B. S. (2015) Polyhydroxyalkanoates: much more than biodegradable plastics. Adv. Appl. Microbiol. 93, 93-106. 
1 (21) Calero, P., and Nikel, P. I. (2018) Chasing bacterial chassis for metabolic engineering: A perspective review from classical to non-traditional microorganisms. Microb. Biotechnol., DOI: 10.1111/1751-7915.13292.

(22) Meng, D. C., Shen, R., Yao, H., Chen, J. C., Wu, Q., and Chen, G. Q. (2014) Engineering the diversity of polyesters. Curr. Opin. Biotechnol. 29, 24-33.

(23) Meng, D. C., and Chen, G. Q. (2018) Synthetic biology of polyhydroxyalkanoates (PHA). Adv. Biochem. Eng. Biotechnol. 162, 147-174.

(24) Leong, Y. K., Show, P. L., Ooi, C. W., Ling, T. C., and Lan, J. C. (2014) Current trends in polyhydroxyalkanoates (PHAs) biosynthesis: insights from the recombinant Escherichia coli. J. Biotechnol. 180, 52-65.

(25) Nikel, P. I., Giordano, A. M., de Almeida, A., Godoy, M. S., and Pettinari, M. J. (2010) Elimination of D-lactate synthesis increases poly(3-hydroxybutyrate) and ethanol synthesis from glycerol and affects cofactor distribution in recombinant Escherichia coli. Appl. Environ. Microbiol. 76 (22), 7400-7406.

(26) Becker, S. H., and Darwin, K. H. (2017) Bacterial proteasomes: Mechanistic and functional insights. Microbiol. Mol. Biol. Rev. 81 (1).

(27) Doma, M. K., and Parker, R. (2007) RNA quality control in eukaryotes. Cell 131 (4), 660-668.

(28) Shoemaker, C. J., Eyler, D. E., and Green, R. (2010) Dom34:Hbs1 promotes subunit dissociation and peptidyl-tRNA drop-off to initiate no-go decay. Science 330 (6002), 369-372.

(29) Carrington, J. C., Cary, S. M., Parks, T. D., and Dougherty, W. G. (1989) A second proteinase encoded by a plant potyvirus genome. EMBO J. 8 (2), 365-370.

(30) Verchot, J., Koonin, E. V., and Carrington, J. C. (1991) The 35-kDa protein from the Nterminus of the potyviral polyprotein functions as a third virus-encoded proteinase. Virology 185 (2), 527-535.

(31) Gorbalenya, A. E., Donchenko, A. P., Blinov, V. M., and Koonin, E. V. (1989) Cysteine proteases of positive strand RNA viruses and chymotrypsin-like serine proteases. A distinct protein superfamily with a common structural fold. FEBS Lett. 243 (2), 103-114. 
1 (32) Kim, D. H., Hwang, D. C., Kang, B. H., Lew, J., and Choi, K. Y. (1996) Characterization of Nla protease from turnip mosaic potyvirus exhibiting a low-temperature optimum catalytic activity. Virology 221 (1), 245-249.

(33) Kim, D. H., Hwang, D. C., Kang, B. H., Lew, J., Han, J., Song, B. D., and Choi, K. Y. (1996) Effects of internal cleavages and mutations in the C-terminal region of Nla protease of turnip mosaic potyvirus on the catalytic activity. Virology 226 (2), 183-190.

(34) Stevens, R. C. (2000) Design of high-throughput methods of protein production for structural biology. Structure 8 (9), R177-R185.

(35) Stein, V., and Alexandrov, K. (2014) Protease-based synthetic sensing and signal amplification. Proc. Natl. Acad. Sci. USA 111 (45), 15934-15939.

(36) Sekar, K., Gentile, A. M., Bostick, J. W., and Tyo, K. E. (2016) N-Terminal-based targeted, inducible protein degradation in Escherichia coli. PLoS One 11 (2), e0149746.

(37) Brockman, I. M., and Prather, K. L. J. (2015) Dynamic knockdown of E. coli central metabolism for redirecting fluxes of primary metabolites. Metab. Eng. 28, 104-113.

(38) Gottesman, S., Roche, E., Zhou, Y., and Sauer, R. T. (1998) The CIpXP and CIpAP proteases degrade proteins with carboxy-terminal peptide tails added by the SsrA-tagging system. Genes Dev. 12 (9), 1338-1347.

(39) Thompson, M. W., Singh, S. K., and Maurizi, M. R. (1994) Processive degradation of proteins by the ATP-dependent Clp protease from Escherichia coli: Requirement for the multiple array of active sites in ClpP but not ATP hydrolysis. J. Biol. Chem. 269 (27), 18209-18215.

(40) Silva-Rocha, R., Martínez-García, E., Calles, B., Chavarría, M., Arce-Rodríguez, A., de las Heras, A., Páez-Espino, A. D., Durante-Rodríguez, G., Kim, J., Nikel, P. I., Platero, R., and de Lorenzo, V. (2013) The Standard European Vector Architecture (SEVA): a coherent platform for the analysis and deployment of complex prokaryotic phenotypes. Nucleic Acids Res. 41 (D1), D666-D675.

(41) Durante-Rodríguez, G., de Lorenzo, V., and Martínez-García, E. (2014) The Standard European Vector Architecture (SEVA) plasmid toolkit. Methods Mol. Biol. 1149, 469-478.

(42) Li, R., Zhang, H., and Qi, Q. (2007) The production of polyhydroxyalkanoates in recombinant Escherichia coli. Bioresour. Technol. 98 (12), 2313-2320. 
1 (43) Egoburo, D. E., Díaz-Peña, R., Álvarez, D. S., Godoy, M. S., Mezzina, M. P., and Pettinari, M. J. (2018) Microbial cell factories à la carte: Elimination of global regulators Cra and ArcA generates metabolic backgrounds suitable for the synthesis of bioproducts in Escherichia coli. Appl. Environ. Microbiol. DOI:10.1128/AEM.01337-18

(44) Li, T., Ye, J., Shen, R., Zong, Y., Zhao, X., Lou, C., and Chen, G. Q. (2016) Semirational approach for ultrahigh poly(3-hydroxybutyrate) accumulation in Escherichia coli by combining one-step library construction and high-throughput screening. ACS Synth. Biol. 5 (11), 13081317.

(45) Anderson, A. J., and Dawes, E. A. (1990) Occurrence, metabolism, metabolic role, and industrial uses of bacterial polyhydroxyalkanoates. Microbiol. Rev. 54 (4), 450-472.

(46) Slater, S., Houmiel, K. L., Tran, M., Mitsky, T. A., Taylor, N. B., Padgette, S. R., and Gruys, K. J. (1998) Multiple b-ketothiolases mediate poly( $\beta$-hydroxyalkanoate) copolymer synthesis in Ralstonia eutropha. J. Bacteriol. 180 (8), 1979-1987.

(47) Chen, G. Q., and Jiang, X. R. (2017) Engineering microorganisms for improving polyhydroxyalkanoate biosynthesis. Curr. Opin. Biotechnol. 53, 20-25.

(48) Hiroe, A., Tsuge, K., Nomura, C. T., Itaya, M., and Tsuge, T. (2012) Rearrangement of gene order in the phaCAB operon leads to effective production of ultrahigh-molecular-weight poly[(R)-3-hydroxybutyrate] in genetically engineered Escherichia coli. Appl. Environ. Microbiol. 78 (9), 3177-3184.

(49) van Wegen, R. J., Lee, S. Y., and Middelberg, A. P. J. (2001) Metabolic and kinetic analysis of poly(3-hydroxybutyrate) production by recombinant Escherichia coli. Biotechnol. Bioeng. $74(1), 70-81$.

(50) Datsenko, K. A., and Wanner, B. L. (2000) One-step inactivation of chromosomal genes in Escherichia coli K-12 using PCR products. Proc. Natl. Acad. Sci. USA 97, 6640-6645.

(51) Vick, J. E., Clomburg, J. M., Blankschien, M. D., Chou, A., Kim, S., and González, R. (2015) Escherichia coli enoyl-acyl carrier protein reductase (Fabl) supports efficient operation of a functional reversal of the $\beta$-oxidation cycle. Appl. Environ. Microbiol. 81 (4), 1406-1416. 
1 (52) Chang, D. E., Shin, S., Rhee, J. S., and Pan, J. G. (1999) Acetate metabolism in a pta mutant of Escherichia coli W3110: Importance of maintaining acetyl coenzyme A flux for growth and survival. J. Bacteriol. 181 (21), 6656-6663.

(53) Hollinshead, W., He, L., and Tang, Y. J. (2014) Biofuel production: an odyssey from metabolic engineering to fermentation scale-up. Front. Microbiol. 5, 344.

(54) Chubukov, V., Mukhopadhyay, A., Petzold, C. J., Keasling, J. D., and García-Martín, H. (2016) Synthetic and systems biology for microbial production of commodity chemicals. Syst. Biol. Appl. 2, 16009.

(55) Natsume, T., and Kanemaki, M. T. (2017) Conditional degrons for controlling protein expression at the protein level. Annu. Rev. Genet. 51 (1), 83-102.

(56) Faden, F., Mielke, S., Lange, D., and Dissmeyer, N. (2014) Generic tools for conditionally altering protein abundance and phenotypes on demand. Biol. Chem. 395 (7-8), 737-762.

(57) Rosano, G. L., and Ceccarelli, E. A. (2014) Recombinant protein expression in Escherichia coli: advances and challenges. Front. Microbiol. 5, 172.

(58) Tate, C. G., Haase, J., Baker, C., Boorsma, M., Magnani, F., Vallis, Y., and Williams, D. C. (2003) Comparison of seven different heterologous protein expression systems for the production of the serotonin transporter. Biochim. Biophys. Acta 1610 (1), 141-153.

(59) Mariscal, A. M., Kakizawa, S., Hsu, J. Y., Tanaka, K., González-González, L., Broto, A., Querol, E., Lluch-Senar, M., Piñero-Lambea, C., Sun, L., Weyman, P. D., Wise, K. S., Merryman, C., Tse, G., Moore, A. J., Hutchison, C. A., Smith, H. O., Tomita, M., Venter, J. C., Glass, J. I., Pinol, J., and Suzuki, Y. (2018) Tuning gene activity by inducible and targeted regulation of gene expression in minimal bacterial cells. ACS Synth. Biol. 7 (6), 1538-1552.

(60) Miller, J. H. (1972) Experiments in molecular genetics. Cold Spring Harbor Laboratory: Cold Spring Harbor, N.Y.

(61) Nikel, P. I., Romero-Campero, F. J., Zeidman, J. A., Goñi-Moreno, A., and de Lorenzo, V. (2015) The glycerol-dependent metabolic persistence of Pseudomonas putida KT2440 reflects the regulatory logic of the GlpR repressor. mBio 6 (2), e00340-00315.

(62) Sambrook, J., and Russell, D. W. (2001) Molecular cloning: a laboratory manual. 3rd ed.; Cold Spring Harbor Laboratory: Cold Spring Harbor. 
(63) Bertram, R., and Hillen, W. (2008) The application of Tet repressor in prokaryotic gene regulation and expression. Microb. Biotechnol. 1 (1), 2-16.

Muthukrishnan, A. B., Kandhavelu, M., Lloyd-Price, J., Kudasov, F., Chowdhury, S., YliHarja, O., and Ribeiro, A. S. (2012) Dynamics of transcription driven by the tetA promoter, one event at a time, in live Escherichia coli cells. Nucleic Acids Res. 40 (17), 8472-8483.

(65) Dvořák, P., Chrást, L., Nikel, P. I., Fedr, R., Soucek, K., Sedlacková, M., Chaloupková, R., de Lorenzo, V., Prokop, Z., and Damborský, J. (2015) Exacerbation of substrate toxicity by IPTG in Escherichia coli BL21(DE3) carrying a synthetic metabolic pathway. Microb. Cell Fact. 14, 201.

(66) Nikel, P. I., and Chavarría, M. (2016) Quantitative physiology approaches to understand and optimize reducing power availability in environmental bacteria. In Hydrocarbon and Lipid Microbiology Protocols-Synthetic and Systems Biology - Tools, McGenity, T. J.; Timmis, K. N.; Nogales-Fernández, B., Eds. Humana Press: Heidelberg, Germany, pp 39-70.

(67) Palmer, M. A. J., Differding, E., Gamboni, R., Williams, S. F., Peoples, O. P., Walsh, C. T., Sinskey, A. J., and Masamune, S. (1991) Biosynthetic thiolase from Zoogloea ramigera. Evidence for a mechanism involving Cys-378 as the active site base. J. Biol. Chem. 266 (13), 8369-8375.

(68) Tyo, K. E., Zhou, H., and Stephanopoulos, G. N. (2006) High-throughput screen for poly-3hydroxybutyrate in Escherichia coli and Synechocystis sp. strain PCC6803. Appl. Environ. Microbiol. 72 (5), 3412-3417.

(69) Martínez-García, E., Aparicio, T., de Lorenzo, V., and Nikel, P. I. (2014) New transposon tools tailored for metabolic engineering of Gram-negative microbial cell factories. Front. Bioeng. Biotechnol. 2, 46.

(70) Nikel, P. I., Pettinari, M. J., Galvagno, M. A., and Méndez, B. S. (2006) Poly(3hydroxybutyrate) synthesis by recombinant Escherichia coli arcA mutants in microaerobiosis. Appl. Environ. Microbiol. 72 (4), 2614-2620.

(71) Ruiz, J. A., Fernández, R. O., Nikel, P. I., Méndez, B. S., and Pettinari, M. J. (2006) dye (arc) Mutants: insights into an unexplained phenotype and its suppression by the synthesis of 
 poly(3-hydroxybutyrate) in Escherichia coli recombinants. FEMS Microbiol. Lett. 258 (1), 55-
(72) Berlanga, M., Montero, M. T., Fernández-Borrell, J., and Guerrero, R. (2006) Rapid spectrofluorometric screening of poly-hydroxyalkanoate-producing bacteria from microbial mats. Int. Microbiol. 9 (2), 95-102.
(73) Pflüger-Grau, K., Chavarría, M., and de Lorenzo, V. (2011) The interplay of the Ell/ ${ }^{\mathrm{Ntr}}$ component of the nitrogen-related phosphotransferase system (PTS Nir) of Pseudomonas putida with pyruvate dehydrogenase. Biochim. Biophys. Acta 1810 (10), 995-1005.
(74) Hanahan, D., and Meselson, M. (1983) Plasmid screening at high colony density. Methods Enzymol. 100, 333-342.
(75) Durfee, T., Nelson, R., Baldwin, S., Plunkett, G., Burland, V., Mau, B., Petrosino, J. F., Qin, X., Muzny, D. M., Ayele, M., Gibbs, R. A., Csörgö, B., Pósfai, G., Weinstock, G. M., and Blattner, F. R. (2008) The complete genome sequence of Escherichia coli DH10B: Insights into the biology of a laboratory workhorse. J. Bacteriol. 190 (7), 2597-2606.
(76) Norrander, J., Kempe, T., and Messing, J. (1983) Construction of improved M13 vectors using oligodeoxynucleotide-directed mutagenesis. Gene 26 (1), 101-106.
(77) Peoples, O. P., and Sinskey, A. J. (1989) Poly- $\beta$-hydroxybutyrate (PHB) biosynthesis in Alcaligenes eutrophus $\mathrm{H} 16$. Identification and characterization of the PHB polymerase gene (phbC). J. Biol. Chem. 264 (26), 15298-15303.




\section{Strain or plasmid \\ Escherichia coli}

Description $^{a}$

\section{TABLES}

$\mathrm{DH} 5 \alpha$

Cloning host; $\mathrm{F}^{-} \lambda^{-}$endA1 gInX44(AS) thiE1 recA1 relA1 spoT1 Hanahan and gyrA96(Nal $\left.{ }^{R}\right) \quad$ rfbC1 deoR nupG $\quad \Phi 80\left(\operatorname{lacZ\Delta M15)} \quad \Delta(\operatorname{argF-lac}) U 169 \quad\right.$ Meselson $^{74}$ $h s d R 17\left(r_{K}^{-} m_{K}^{+}\right)$

DH10B Cloning host; $\mathrm{F}^{-} \lambda^{-}$endA1 recA1 galK galU $\Delta\left(\right.$ ara-leu)7697 araD139 deoR Durfee et al. ${ }^{75}$ nupG rpsL $\Phi 80($ lacZ $\Delta M 15)$ mcrA $\Delta(m r r-h s d R M S-m c r B C) \Delta / a c X 74$

BW25113

Wild-type strain; $\mathrm{F}^{-} \lambda^{-} \Delta(\mathrm{araD}$-araB) $567 \Delta$ lacZ4787(::rrnB-3) rph-1 $\Delta($ rhaD- Datsenko and rhaB)568 hsdR514

Wanner ${ }^{50}$

\section{Plasmids}

pSEVA238

Expression vector; oriV(pBBR1), XyIS/Pm expression system; $\mathrm{Km}^{\mathrm{R}}$

Silva-Rocha et al. ${ }^{40}$

pSEVA637

Cloning vector; oriV(pBBR1), promoter-less GFP; $\mathrm{Gm}^{\mathrm{R}}$

Silva-Rocha

pSEVA237R Cloning vector; oriV(pBBR1), promoter-less mCherry; $\mathrm{Km}^{\mathrm{R}}$ et al. ${ }^{40}$

Silva-Rocha

pSEVA341 Cloning vector; oriV(pR01600/ColE1); $\mathrm{Cm}^{\mathrm{R}}$ et al. ${ }^{40}$

Silva-Rocha

$\mathrm{pS} 238 \cdot \mathrm{Nla}$

Derivative of vector pSEVA238 used for regulated expression of nia, et al. ${ }^{40}$

pS341T encoding the potyvirus Nla protease; $\mathrm{XyIS/Pm} \rightarrow$ nia; $\mathrm{Km}^{\mathrm{R}}$

pS341T·gfp

Derivative of vector pSEVA341 carrying the constitutive $\mathrm{P}_{\text {tetA }}$ promoter; $\mathrm{Cm}^{\mathrm{R}}$ This work Derivative of vector pSEVA341T used for constitutive expression of $g f p$; This work $\mathrm{P}_{\text {tetA }} \rightarrow g f p ; \mathrm{Cm}^{\mathrm{R}}$ 

$p S 341 T \cdot g f p^{* b} \quad$ Derivative of vector pSEVA341T used for constitutive expression of a This work variant of $g f p\left(g f p^{*}\right) ; P_{t e t A} \rightarrow g f p^{*} ; \mathrm{Cm}^{R}$

\begin{tabular}{|c|c|c|}
\hline$r y$ & $\begin{array}{l}\text { Derivative of vector pSEVA341T used for constitutive expression of } \\
m C h e r r y ; \mathrm{P}_{\text {tetA }} \rightarrow m C h e r r y ; \mathrm{Cm}^{\mathrm{R}}\end{array}$ & his work \\
\hline $\mathrm{pS} 341 \mathrm{~T} \cdot \mathrm{mCherry}{ }^{*}$ & $\begin{array}{l}\text { Derivative of vector pSEVA341T used for constitutive expression of a } \\
\text { variant of } m \text { Cherry }\left(m C h e r r y{ }^{*}\right) ; P_{\text {tetA }} \rightarrow m C h e r r y * ; \mathrm{Cm}^{R}\end{array}$ & This work \\
\hline pFENI) & $\begin{array}{l}\text { Derivative of plasmid } p S 341 \mathrm{~T} \cdot g f p^{*} \text { in which gfp has been tagged with nia } \\
\text { and } s s r A \text { recognition targets; } \mathrm{Cm}^{R}\end{array}$ & This work \\
\hline pFENIX·mCherry ${ }^{*}$ & $\begin{array}{l}\text { Derivative of plasmid } \mathrm{pS} 341 \mathrm{~T} \cdot \mathrm{mCherry} \text { in which } m \text { Cherry has been tagged } \\
\text { with nia and } s s r A \text { recognition targets; } \mathrm{Cm}^{\mathrm{R}}\end{array}$ & This work \\
\hline pAeT41 & $\begin{array}{l}\text { Derivative of vector pUC18 }{ }^{76} \text { bearing a ca. } 5-\mathrm{kb} \text { Smal/EcoRI DNA fragment } \\
\text { from Cupriavidus necator spanning the phaC1AB1 gene cluster; } A p^{R}\end{array}$ & $\begin{array}{l}\text { Peoples and } \\
\text { Sinskey }{ }^{77}\end{array}$ \\
\hline pAeT41·PHA* & $\begin{array}{l}\text { Derivative of plasmid } \mathrm{pAeT} 41 \text { in which phaA has been tagged with nia and } \\
s s r A \text { recognition targets; } A p^{R}\end{array}$ & work \\
\hline pS341.PHA & Derivative of vector pSEVA341 carrying the phaC1AB1 gene cluster; $\mathrm{Cm}^{\mathrm{R}}$ & This work \\
\hline pFEl & $\begin{array}{l}\text { Derivative of vector pSEVA341 in which phaA has been tagged with nia and } \\
\text { ssrA recognition targets; } \mathrm{Cm}^{\mathrm{R}}\end{array}$ & This work \\
\hline
\end{tabular}

1

2 a Antibiotic markers: Ap, ampicillin; Cm, chloramphenicol; Gm, gentamycin; Km, kanamycin; Nal, 3 nalidixic acid.

4 b Modified variants of the GFP and mCherry fluorescent proteins were designed to have exactly

5 the same amino acid sequence as the proteolizable versions after the action of the Nla protease.

6 These variants are indicated by an asterisk $\left({ }^{*}\right)$ symbol as they display the same amino acid

7 sequence as the FENIX-tagged proteins. 
FIG. 1. Rationale and construction of the FENIX system. (a) Nla- and SsrA-dependent posttranslational control of target proteins with the FENIX system. The gene encoding the target polypeptide is added with a synthetic, hybrid nia/ssrA tag, resulting in a tagged protein in which the C-terminus displays the GESNVVVHQADER AANDENYALAA amino acid sequence. The SsrA tag is directly recognized by the CIpXP and ClpAP proteases of the bacterial proteasome in vivo, thus degrading the protein. Upon action of the specific potyvirus Nla protease (the recognition site in the synthetic nia/ssrA tag is indicated with an inverted red triangle in the diagram), the SsrA tag is released and the polypeptide can be accumulated. (b) pFENIX plasmids for one-step cloning and tagging of individual target proteins. The gene encoding the target polypeptide (gene of interest, goi) is amplified by PCR with specific oligonucleotides that include Nhel and BsrGI restriction sites. The resulting amplicon can be directly cloned into plasmid pFENIX·gfp* (which contains a nia/ssrA tagged

\section{FIG. 2. Evaluation of the FENIX system in recombinant $E$. coli using fluorescent proteins.} Plasmids pFENIX·gfp* and pFENIX·mCherry*, which contain the nia/ssrA-tagged versions of the fluorescent proteins (indicated with blue and orange strips, respectively, in the first row of the table), were transformed into E. coli DH10B carrying either plasmid pS238.Nla or the empty pSEVA238 vector (indicated as + and - , respectively, in the second row of the table). In the first four columns of each experiment, the cells contained the nia/ssrA-tagged fluorescent protein, whereas the last two columns represent a negative and positive control, respectively. These control experiments were carried out with $E$. coli DH10B transformed either with the empty pFENIX vector (i.e. no fluorescent protein) or with a plasmid constitutively expressing the gene encoding each fluorescent protein (pS341T·gfp* or pS341T·mCherry ${ }^{*}$, see Table 1). Multi-well microtiter plates containing LB medium with the necessary antibiotics and additives $(1 \mathrm{mM}$ 3-methylbenzoate as the inducer of nia expression, as indicated in the third row of the table), were inoculated with a culture of the 
1 corresponding strain previously grown overnight in LB medium with the necessary antibiotics. Cells

2 were incubated at $37^{\circ} \mathrm{C}$ with rotary agitation, and fluorescence and bacterial growth (expressed as

3 the optical density measured at $600 \mathrm{~nm}, \mathrm{OD}_{600}$ ) were recorded after $24 \mathrm{~h}$. The specific (Sp) activity of

4 the fluorescent proteins under study was calculated as the arbitrary fluorescence units (a.f.u.)

5 normalized to the $\mathrm{OD}_{600}$. Each bar represents the mean value of the $\mathrm{Sp}$ activity \pm standard deviation

6 calculated from at least three independent experiments. The lower panel shows bacterial pellets

7 harvested from shake-flask cultures after $24 \mathrm{~h}$ of incubation under the same growth conditions

8 indicated for the microtiter-plate cultures as observed under blue light.

9

FIG. 3. Flow cytometry analysis of the FENIX system. (a) Time-lapse flow cytometry analysis of

11 GFP* fluorescence (in arbitrary units, a.u.) in shake-flask cultures of $E$. coli DH10B carrying the 12 plasmids indicated. Cells were grown in LB medium at $37^{\circ} \mathrm{C}$ with rotary agitation with the appropriate 13 antibiotics and additives explained in the Methods section, and samples were taken at selected times 14 post-induction $\left(t_{P I}\right)$. The induction of the FENIX system was achieved by addition of 3-methylbenzoate $15(3-\mathrm{mBz})$ to the cultures at $1 \mathrm{mM}$ at the onset of the cultivation. The light grey rectangle in each 16 histogram plot identifies the region considered negative for the fluorescence signal (as assessed with 17 cells carrying plasmid pS238-Nla). The structure of the nia/ssrA-tagged GFP and variants thereof is 18 schematically shown in the last panel (the blue and orange strips represent the Nla and SsrA tags, 19 respectively) along with the Nla protease (in yellow). Note that a modified version of GFP, displaying 20 exactly the same amino acid sequence of GFP* after proteolysis, has been used as a positive control 21 (ctrl.). (b) Induction levels of the FENIX system as calculated from flow cytometry experiments. FIG. 4. Rationale of the FENIX-based metabolic switch designed for controlled biopolymer accumulation in recombinant $\boldsymbol{E}$. coli strains. (a) Poly(3-hydroxybutyrate) (PHB) biosynthesis pathway. Three enzymes are necessary for the de novo biosynthesis of PHB in Cupriavidus necator: 3-ketoacyl-coenzyme A (CoA) thiolase (PhaA, key step of the route as highlighted in the scheme), NADPH-dependent 3-acetoacetyl-CoA reductase (PhaB1), and PHA synthase (PhaC1). PhaA and PhaB1 catalyze the condensation of two molecules of acetyl-CoA to 3-acetoacetyl-CoA and the reduction of acetoacetyl-CoA to $R$-(-)-3-hydroxybutyryl-CoA, respectively. PhaC1 polymerizes the 
1 resulting $\mathrm{C} 4$ monomers into $\mathrm{PHB}$, whereas one $\mathrm{CoA}-\mathrm{SH}$ molecule is released per monomer. $\mathrm{PHB}$ is

2 stored as water-insoluble granules in the cytoplasm of the cells. (b) Synthetic circuit based on the

3 FENIX system for controlled PHB accumulation. PhaA has been earmarked with the synthetic

$4 \mathrm{Nla} / \mathrm{SsrA}$ tag in the C-terminal domain $\left(\mathrm{PhaA}^{*}\right)$, thus rendering the polypeptide susceptible to

5 proteolysis by the bacterial proteasome. Under these circumstances, no PHB is accumulated by the cells. Upon activation of the Nla protease (from a separate plasmid, in which the XyIS/Pm-dependent

7 expression of nia can be triggered by addition of 3-methylbenzoate to the culture medium), the SsrA

8 tag is removed from the protein, the active PhaA enzyme accumulates in the cells and so does PHB.

9 The genetic elements in this scheme are not drawn to scale.

FIG. 5. Physiological and biochemical characterization of $E$. coli strains carrying the FENIX system tailored for controlled PHB accumulation. (a) In vitro determination of the specific (Sp) 3ketoacyl-coenzyme A thiolase (PhaA) activity. E. coli BW25113 was transformed both with plasmids pS238. Nla and pFENIX.PHA* (the structure of the nia/ssrA-tagged variant of phaA in the phaC1AB1 gene cluster of $C$. necator is schematically shown in the upper part of the figure), and the PhaA activity was periodically determined in cell-free extracts as detailed in Methods. The inverted red triangle indicates the addition of 3-methylbenzoate (3-mBz) at $1 \mathrm{mM}$ to the culture medium; the gray bar identifies the maximum thiolase activity detected in E. coli BW25113 transformed only with plasmid pS238-Nla. (b) In vitro determination of the Sp PhaA activity and (c) PHB accumulation in E. coli BW25113 carrying vector pS238.Nla and the indicated plasmids. Plasmids pAeT41 and pS341.PHA express the native phaC1AB1 gene cluster of $C$. necator in different backbones, and the origin of replication of these vectors have a similar copy number (both are variants of pMB1). In all plasmids used in these experiments, the expression of the pha gene cluster is driven by the native, constitutive $P_{\text {pha }}$ promoter. All shake-flask cultures shown in this figure were carried out in LB medium added with $30 \mathrm{~g} \mathrm{~L}^{-1}$ glucose and the adequate antibiotics and additives specified in Methods. Each parameter is reported as the mean value \pm standard deviation from duplicate measurements in at least three independent experiments. Significant differences $(P<0.05$, as evaluated by means of the Student's $t$ test) in the pair-wise comparison of induced versus non-induced cultures are indicated by the $\uparrow$ symbol. 
2 FIG. 6. Growth and PHB accumulation by recombinant E. coli carrying PhaA*. (a) Bacterial 3 growth, expressed as the density of cell dry weight, and (b) PHB content on biomass in shake-flask 4 cultures of $E$. coli BW25113/pS238-Nla transformed either with plasmid pS341·PHA (expressing the 5 native pha gene cluster, identified as PhaA) or pFENIX·PHA* (expressing the nia/ssrA-tagged variant 6 of phaA, identified as $\mathrm{PhaA}^{*}$ ). The inverted red triangle indicates the addition of 3-methylbenzoate (3$7 \mathrm{mBz}$ ) at $1 \mathrm{mM}$ to the culture medium (M9 minimal medium containing $30 \mathrm{~g} \mathrm{~L}^{-1}$ glucose); the gray bar 8 also identifies the time pre-induction of the system. (c) Specific rates of bacterial growth $(\mu)$ and PHB 9 accumulation $\left(r_{\mathrm{PHB}}\right)$ in the strains under study. Significant differences $(P<0.05$, as evaluated by means of the Student's $t$ test) in the pair-wise comparison between the two strains is indicated by the

$11 \dagger$ symbol. In the graphics (a-c), each parameter is reported as the mean value \pm standard deviation 12 from duplicate measurements in at least three independent experiments. (d) Qualitative assessment 13 of PHB accumulation in samples taken from shake-flask cultures at the indicated times and stained 14 with the lipophilic Nile Red dye. Stained cells were observed under the microscope either under 15 phase contrast or fluorescence as indicated in Methods.

FIG. 7. Establishing an orthogonal metabolic switch at the acetyl-CoA node based on the FENIX system. (a) The acetyl-coenzyme A (CoA) metabolic node in the recombinant $E$. coli strains used in this study. The wide shaded arrow represents the central pathways leading to acetyl-CoA formation (i.e. glycolysis); this intermediate is used as a precursor in a myriad of metabolic reactions

21 (not indicated in the scheme). The main sinks of acetyl-CoA are shown, namely, PHB biosynthesis or acetate formation (catalyzed by Pta, phosphotransacetylase, and AckA, acetate kinase). The Nla protease of the FENIX system, mediating the metabolic switch, is indicated in yellow. (b) Specific rate of acetate formation, as determined by secretion of acetate into the culture medium during exponential growth. (c) Intracellular content of acetyl-CoA, evaluated by LC-MS in cell-free extracts as explained in Methods, during mid-exponential growth. All shake-flask cultures shown in this figure were carried out in M9 minimal medium added with $30 \mathrm{~g} \mathrm{~L}^{-1}$ glucose and the adequate antibiotics and additives specified in Methods. E. coli BW25113 was transformed with plasmid pS238.Nla in all cases. Each parameter is reported as the mean value \pm standard deviation from duplicate 
1 measurements in at least two independent experiments. Significant differences $(P<0.05$, as 2 evaluated by means of the Student's $t$ test) in the pair-wise comparison of each recombinant strain 3 against the control strain (carrying the empty pSEVA341 vector) are indicated by the $\uparrow$ symbol. $3-H B-$ 4 CoA, R-(-)-3-hydroxybutyryl-CoA; CDW, cell dry weight. 


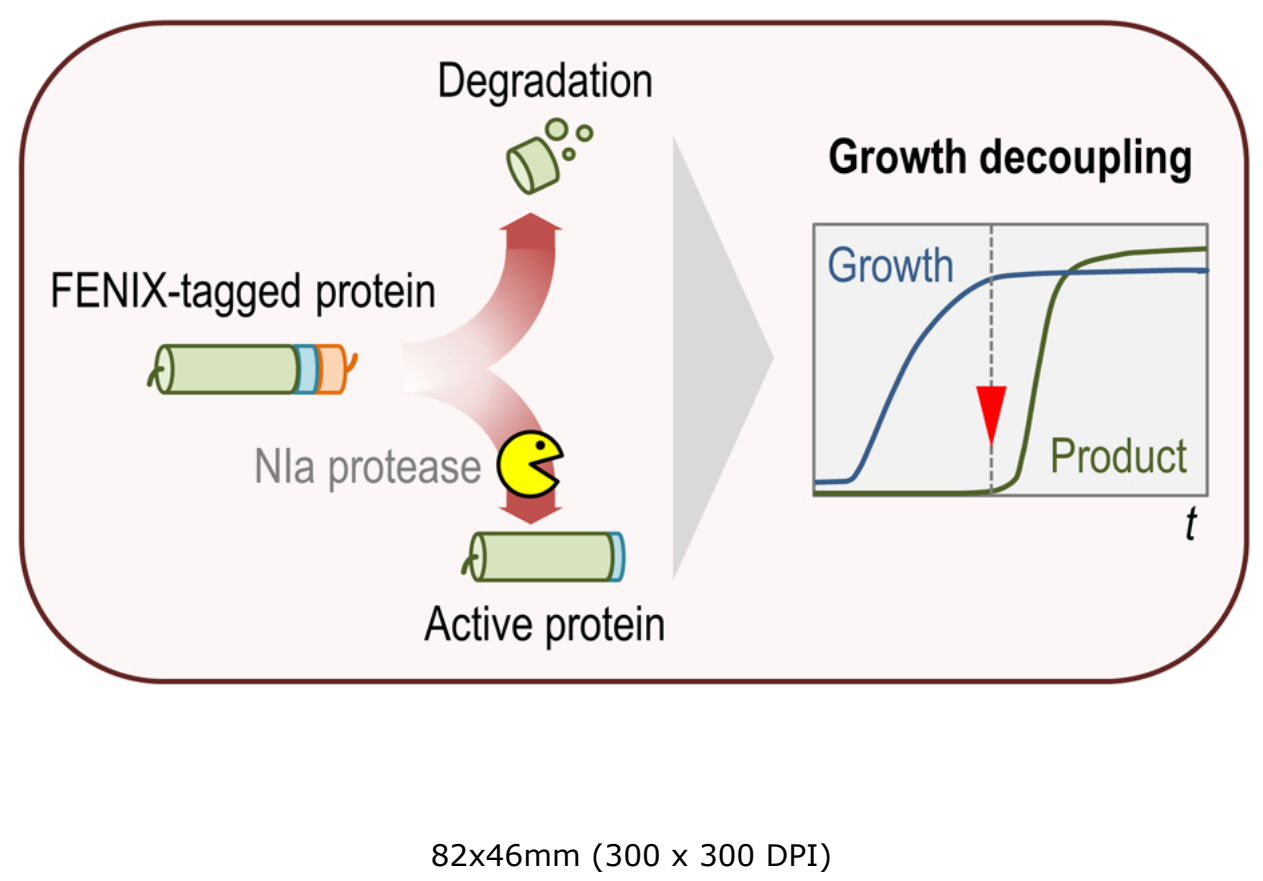

ACS Paragon Plus Environment 
(a)
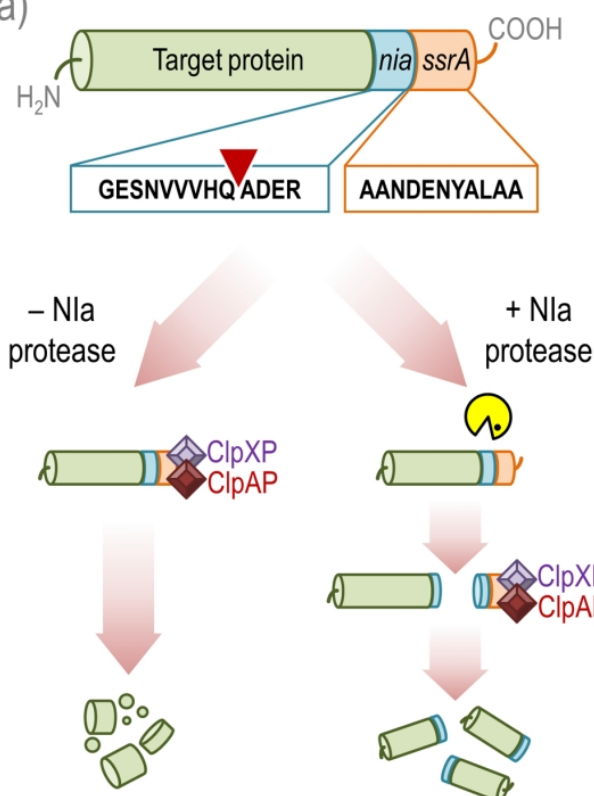

Protein degradation

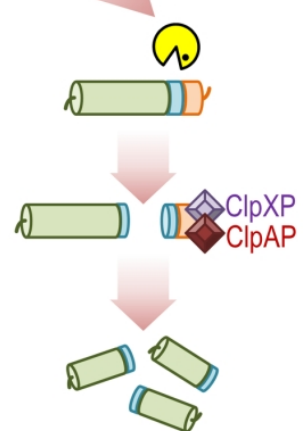

Active protein (b)

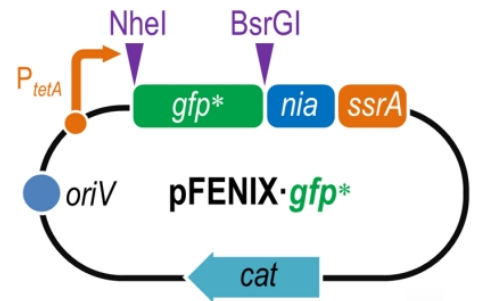

5'-Nhel- -

\section{Gene of interest}

PCR amplification

Digestion with Nhel and BsrGI and ligation

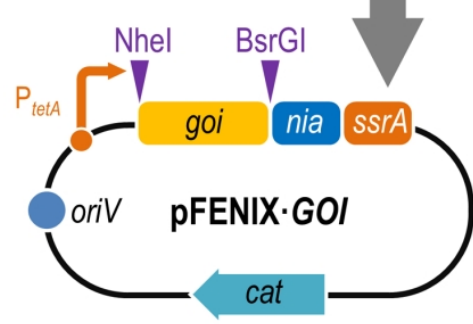

$170 \times 122 \mathrm{~mm}(300 \times 300 \mathrm{DPI})$ 


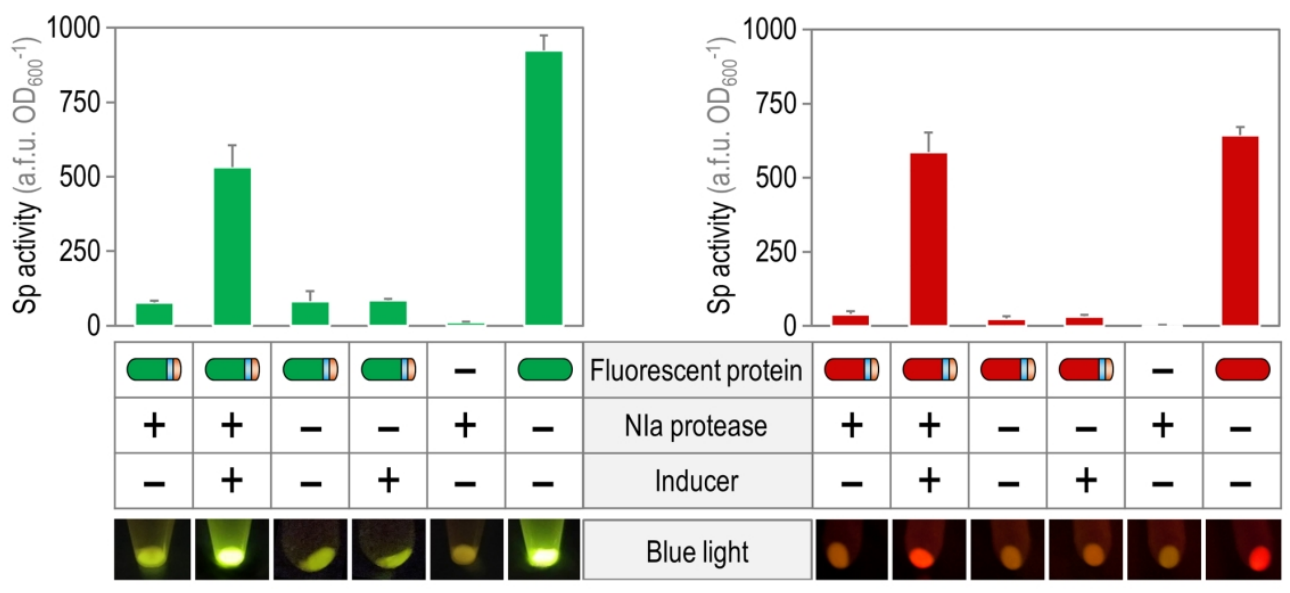

$170 \times 76 \mathrm{~mm}(300 \times 300$ DPI $)$ 

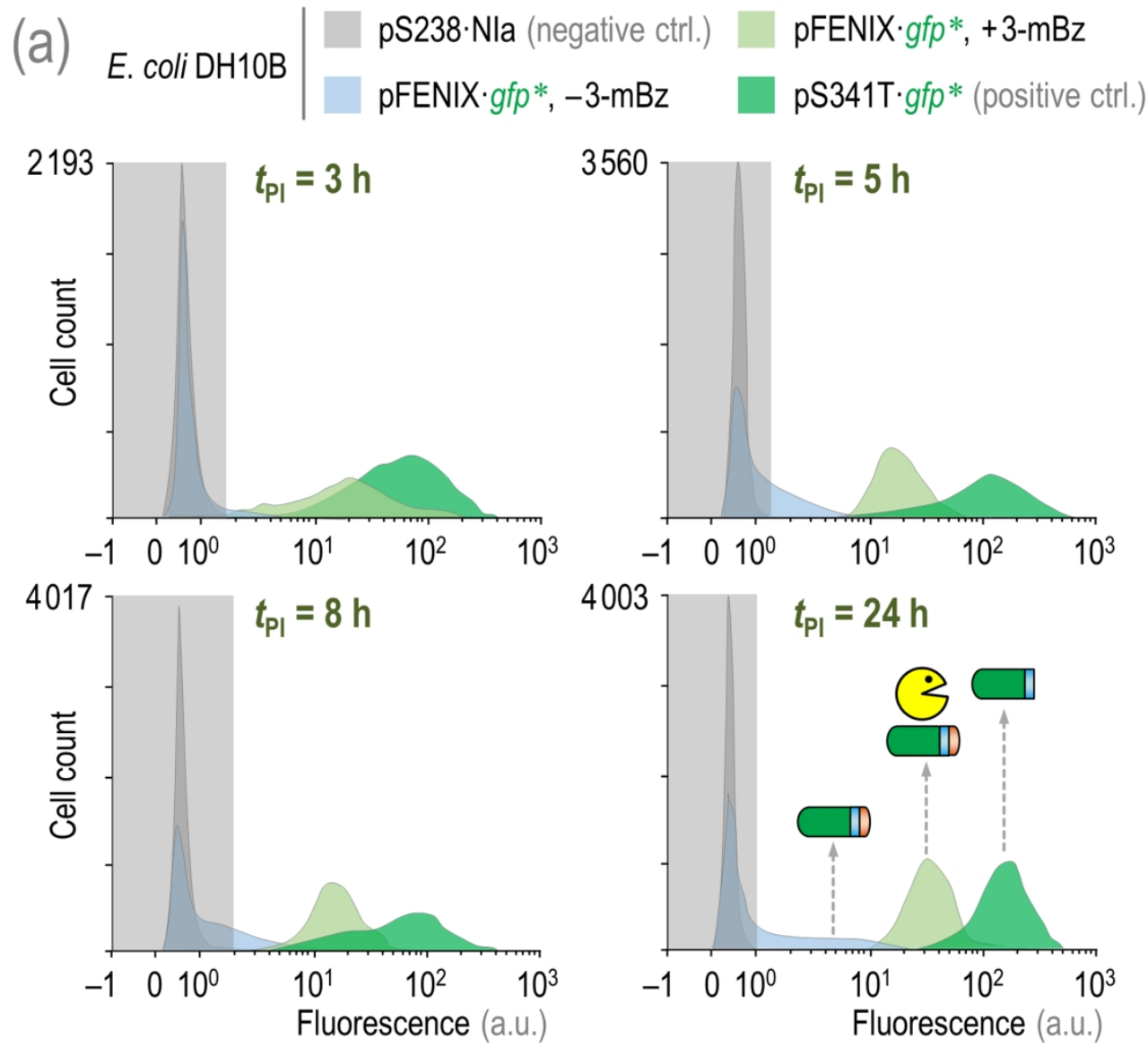

(b)

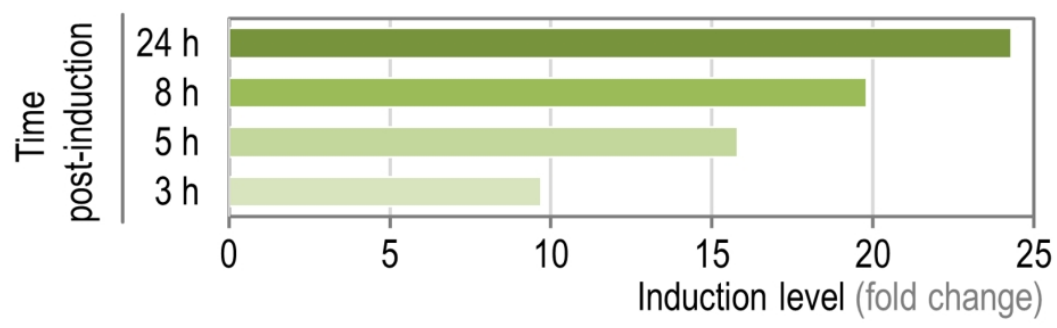

$170 \times 199 \mathrm{~mm}(300 \times 300$ DPI $)$ 
(a)

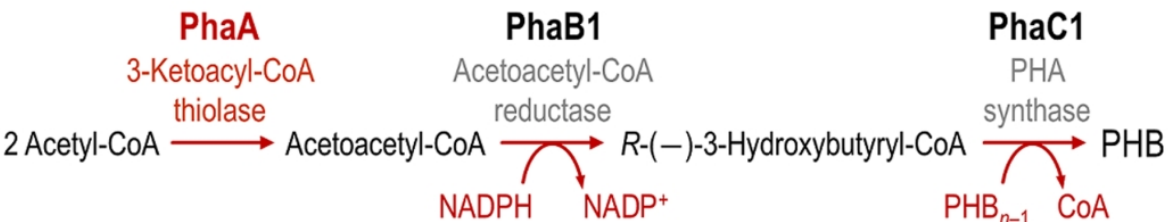

(b)

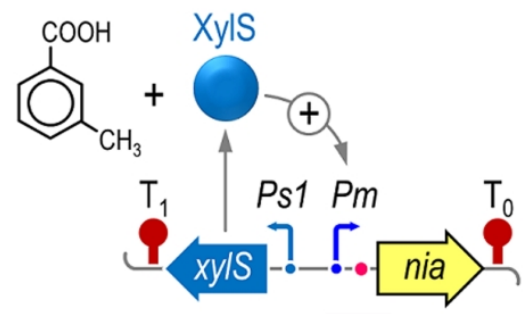

Active PhaA
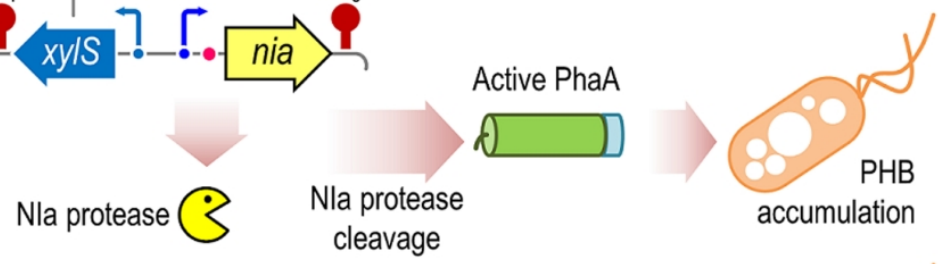

accumulation
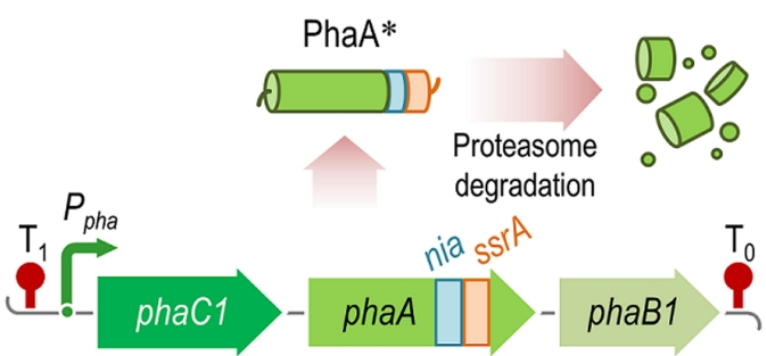

$170 \times 149 \mathrm{~mm}(300 \times 300 \mathrm{DPI})$

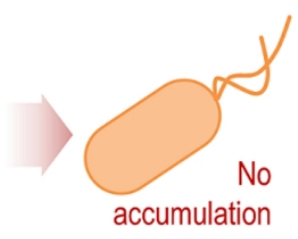

30 

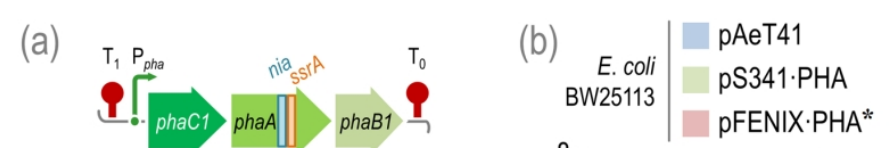

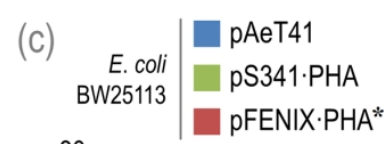
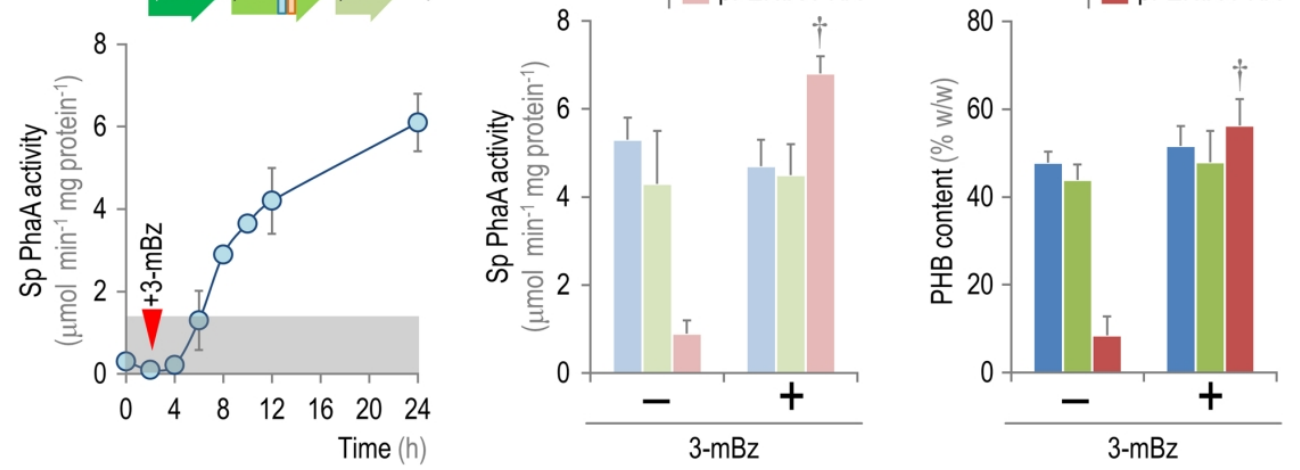

$170 \times 79 \mathrm{~mm}(300 \times 300 \mathrm{DPI})$ 

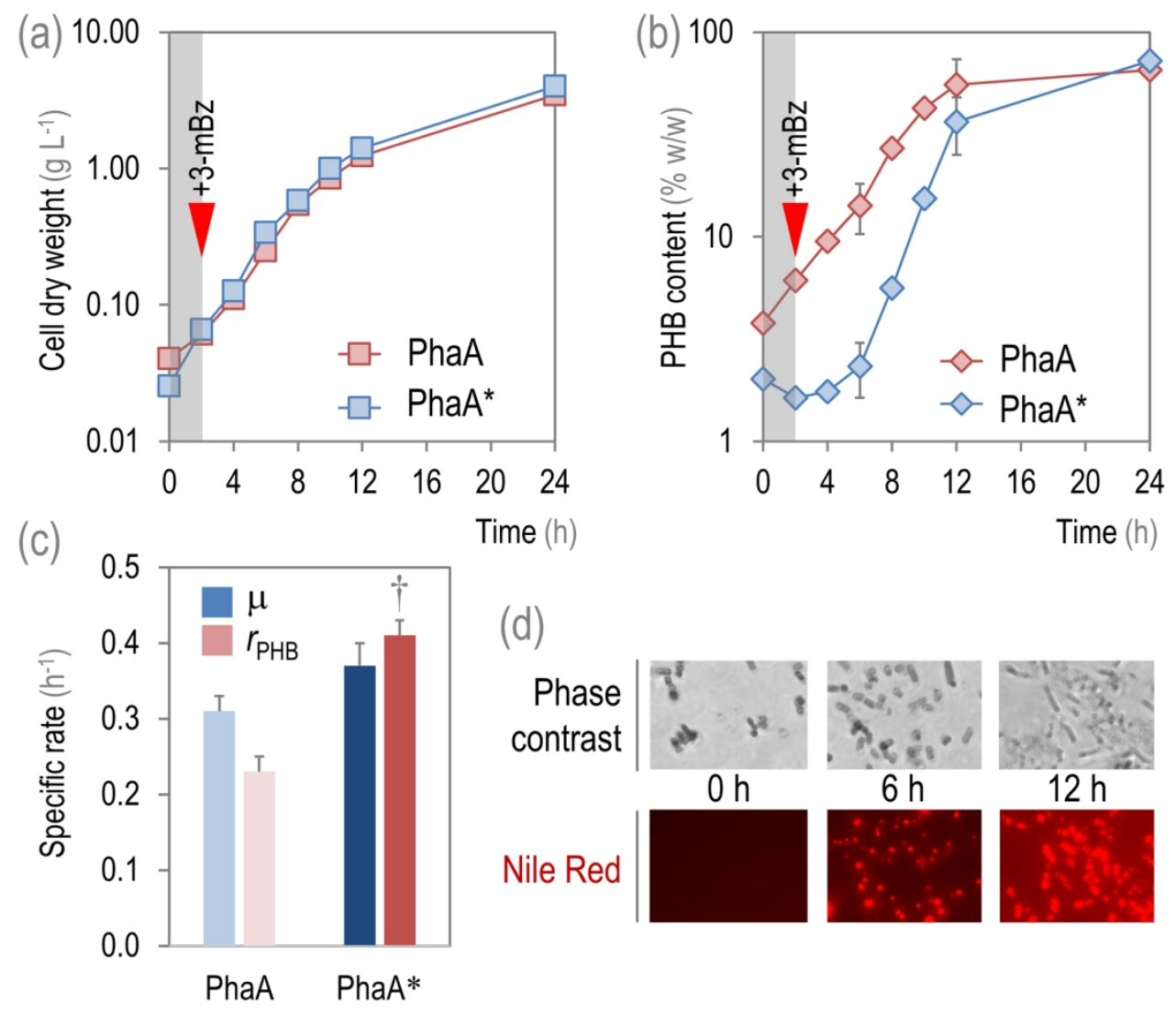

$170 \times 143 \mathrm{~mm}(300 \times 300 \mathrm{DPI})$ 
(a)

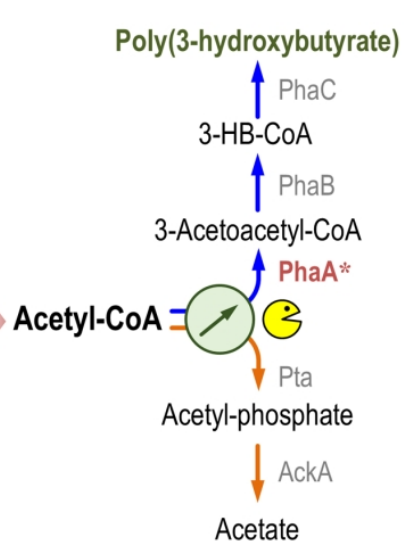

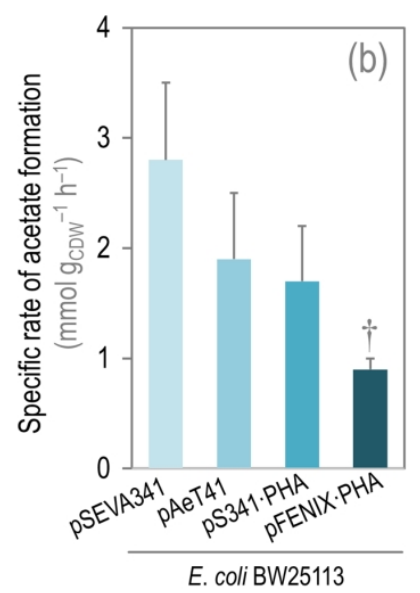

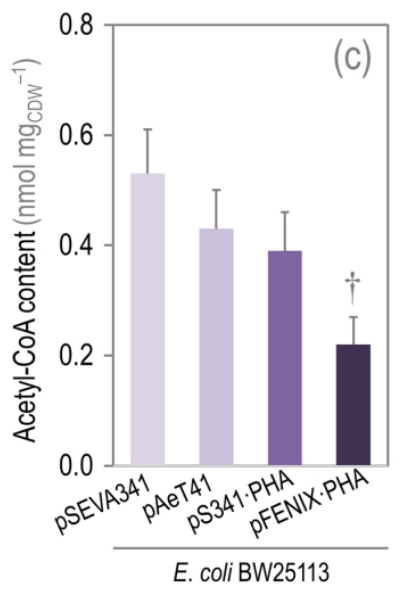

$170 \times 77 \mathrm{~mm}(300 \times 300 \mathrm{DPI})$ 